\title{
SPECTROSCOPIC STUDIES OF EXTREMELY METAL-POOR STARS WITH THE SUBARU HIGH DISPERSION SPECTROGRAPH. I. OBSERVATIONAL DATA ${ }^{1}$
}

\author{
Satoshi Honda, ${ }^{2,3}$ Wako Aoki, ${ }^{2,3}$ Hiroyasu Ando, ${ }^{2}$ Hideyuki Izumiura, ${ }^{4}$ Toshitaka Kajino, ${ }^{2,3}$ Eiji Kambe, ${ }^{5}$ \\ Satoshi Kawanomoto, ${ }^{2}$ Kunio Noguchi, ${ }^{2,3}$ KiIchi Okita, ${ }^{4}$ Kozo Sadakane, ${ }^{6}$ Bun’ei Sato, ${ }^{2,7}$ \\ Masahide Takada-Hidai, ${ }^{8}$ Yoichi Takeda, ${ }^{2,9}$ Etsuji Watanabe, ${ }^{4}$ Timothy C. Beers, ${ }^{10}$ \\ John E. Norris, ${ }^{11}$ and SEAN G. Ryan ${ }^{12}$ \\ Received 2003 July 10; accepted 2004 January 27
}

\begin{abstract}
We have obtained high-resolution $(R \simeq 50,000$ or 90,000$)$, high-quality ( $\mathrm{S} / \mathrm{N} \gtrsim 100)$ spectra of 22 very metalpoor stars $([\mathrm{Fe} / \mathrm{H}] \lesssim-2.5)$ with the High Dispersion Spectrograph fabricated for the $8.2 \mathrm{~m}$ Subaru Telescope. The spectra cover the wavelength range from 3500 to $5100 \AA$; equivalent widths are measured for isolated lines of numerous elemental species, including the $\alpha$-elements, the iron-peak elements, and the light and heavy neutron-capture elements. Errors in the measurements and comparisons with previous studies are discussed. These data will be used to perform detailed abundance analyses in the following papers of this series. Radial velocities are also reported and are compared with previous studies. At least one moderately $r$-process-enhanced metal-poor star, HD 186478, exhibits evidence of a small-amplitude radial velocity variation, confirming the binary status noted previously. During the course of this initial program, we have discovered a new moderately $r$-process-enhanced, very metal-poor star, CS 30306-132 $([\mathrm{Fe} / \mathrm{H}]=-2.4 ;[\mathrm{Eu} / \mathrm{Fe}]=+0.85)$, which is discussed in detail in the companion paper.
\end{abstract}

Subject headings: nuclear reactions, nucleosynthesis, abundances — stars: abundances — stars: Population II On-line material: machine-readable table

\section{INTRODUCTION}

Very metal-poor stars $([\mathrm{Fe} / \mathrm{H}] \lesssim-2.5)^{13}$ are believed to have been born in the early Galaxy; their chemical compositions are living records of the nucleosynthesis processes that preceded their formation. As a result of considerable efforts

\footnotetext{
${ }^{1}$ Based on data collected at Subaru Telescope, which is operated by the National Astronomical Observatory of Japan.

${ }^{2}$ National Astronomical Observatory, Mitaka, Tokyo 181-8588, Japan; honda@optik.mtk.nao.ac.jp,aoki.wako@nao.ac.jp,ando@optik.mtk.nao.ac.jp, kajino@nao.ac.jp, kawanomo@optik.mtk.nao.ac.jp, knoguchi@optik.mtk.nao .ac.jp.

3 Department of Astronomy, Graduate University for Advanced Studies, Mitaka, Tokyo 181-8588, Japan.

${ }^{4}$ Okayama Astrophysical Observatory, National Astronomical Observatory of Japan, Kamogata-cho, Okayama 719-0232, Japan; izumiura@oao.nao .ac.jp, okita@oao.nao.ac.jp, watanabe@oao.nao.ac.jp.

5 Department of Earth and Ocean Sciences, National Defense Academy, Hashirimizu 1-10-20, Yokosuka, Kanagawa 239-8686, Japan; kambe@nda .ac.jp.

6 Astronomical Institute, Osaka Kyoiku University, Kashiwara-shi, Osaka 582-8582, Japan; sadakane@cc.osaka-kyoiku.ac.jp.

${ }^{7}$ Department of Astronomy, School of Science, University of Tokyo, Bunkyo-ku, Tokyo 113-0033, Japan; satobn@oao.nao.ac.jp.

${ }^{8}$ Liberal Arts Education Center, Tokai University, 1117 Kitakaname, Hiratsuka-shi, Kanagawa 259-1292, Japan; hidai@apus.rh.u-tokai.ac.jp.

${ }^{9}$ Komazawa University, Komazawa, Setagaya, Tokyo 154-8525, Japan; takedayi@cc.nao.ac.jp.

${ }^{10}$ Department of Physics and Astronomy, Michigan State University, East Lansing, MI 48824-1116; beers@pa.msu.edu.

${ }_{11}$ Reseach School of Astronomy and Astrophysics, Australian National University, Private Bag, Ewston Creek Post Office, Canberra, ACT 2611, Australia; jen@mso.anu.edu.au.

12 Department of Physics and Astronomy, Open University, Walton Hall, Milton Keynes MK7 6AA, UK; s.g.ryan@open.ac.uk.

13 We use the usual notation $[\mathrm{A} / \mathrm{B}] \equiv \log _{10}\left(N_{\mathrm{A}} / N_{\mathrm{B}}\right)_{*}-\log _{10}\left(N_{\mathrm{A}} / N_{\mathrm{B}}\right)$. and $\log \epsilon(\mathrm{A}) \equiv \log _{10}\left(N_{\mathrm{A}} / N_{\mathrm{H}}\right)+12.0$, for elements $\mathrm{A}$ and $\mathrm{B}$. Also, the term "metallicity" will be assumed here to be equivalent to the stellar $[\mathrm{Fe} / \mathrm{H}]$ value.
}

by many astronomers, a large list of candidate stars with $[\mathrm{Fe} / \mathrm{H}]<-2.5$ have been provided by wide-field objectiveprism surveys in the past two decades (e.g., the HK survey: Beers, Preston, \& Shectman 1985, 1992, Beers 1999; and the Hamburg/ESO Survey: Christlieb \& Beers 2000, Christlieb et al. 2001, Christlieb 2003). Over the past several years, highresolution spectroscopic studies have enabled the measurement of elemental abundances for many of the metal-poor stars found by these surveys (e.g., McWilliam et al. 1995a; Ryan, Norris, \& Beers 1996; Burris et al. 2000; Carretta et al. 2002; Cayrel et al. 2004), including detailed studies of the lowest metallicity stars yet identified (e.g., Norris, Ryan, \& Beers 2001; Christlieb et al. 2002). These observational studies, which continue at present, are providing strong constraints on models of the dominant nucleosynthesis processes in the earliest epochs of star formation in our Galaxy, in particular those associated with massive stars and Type II supernovae.

Remarkable progress has been made, in particular, through studies of the neutron-capture elements in very metal-poor stars. High-resolution spectroscopic studies of very metalpoor stars have revealed, for example, that a small fraction (presently estimated to be on the order of $2 \%-3 \%, \mathrm{~T}$. C. Beers 2003 , private communication) of giants with $[\mathrm{Fe} / \mathrm{H}]<-2.5$ exhibit large overabundances (e.g., $[r$-process $/ \mathrm{Fe}]>+1.0$ ) of neutron-capture elements associated with the $r$-process (e.g., $[r$-process $/ \mathrm{Fe}]>+1.0 ;$ McWilliam et al. 1995a; Sneden et al. 2000, 2003; Cayrel et al. 2001; Hill et al. 2002). These, along with a handful of other metal-poor stars with moderately enhanced $r$-process elements $(+0.5 \leq[r$-process $/ \mathrm{Fe}] \leq+1.0$, e.g., Westin et al. 2000; Johnson \& Bolte 2001; Cowan et al. 2002), display remarkably similar abundance patterns in the range $56 \leq Z<76$, all apparently in good agreement with the solar-system $r$-process component. In addition, some of 
TABLE 1

Program Stars and Observation Log

\begin{tabular}{|c|c|c|c|c|c|c|c|}
\hline Number & Star & $V$ & $B-V$ & $R$ & $\mathrm{~S} / \mathrm{N}^{\mathrm{a}}$ & $\mathrm{S} / \mathrm{N}^{\mathrm{b}}$ & $\begin{array}{c}\text { Exposure } \\
\text { (s) }\end{array}$ \\
\hline 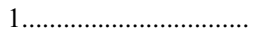 & HD 4306 & 9.08 & 0.63 & 90000 & 272 & 497 & 1800 \\
\hline $2 \ldots \ldots \ldots \ldots \ldots \ldots \ldots \ldots$ & HD 6268 & 8.10 & 0.79 & 90000 & 158 & 288 & 1800 \\
\hline 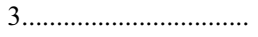 & HD 88609 & 8.59 & 0.93 & 90000 & 62 & 113 & 2110 \\
\hline $4 \ldots \ldots \ldots \ldots \ldots \ldots \ldots . .$. & HD 110184 & 8.31 & 1.17 & 90000 & 221 & 403 & 900 \\
\hline 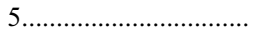 & HD 115444 & 8.97 & 0.78 & 90000 & 255 & 466 & 3900 \\
\hline 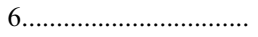 & HD 122563 & 6.20 & 0.91 & 90000 & 374 & 683 & 1200 \\
\hline 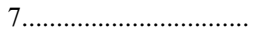 & HD 126587 & 9.15 & 0.73 & 90000 & 187 & 341 & 4500 \\
\hline 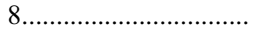 & HD 140283 & 7.21 & 0.49 & 90000 & 458 & 836 & 3600 \\
\hline 9 & HD 186478 & 9.18 & 0.90 & 100000 & 158 & 274 & 2400 \\
\hline $10 \ldots \ldots \ldots \ldots \ldots \ldots \ldots$ & BS $16082-129$ & 13.55 & 0.67 & 50000 & 55 & 135 & 5400 \\
\hline 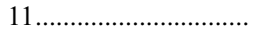 & BS $16085-050$ & 12.15 & 0.74 & 50000 & 100 & 245 & 5100 \\
\hline $12 \ldots \ldots \ldots \ldots \ldots \ldots \ldots \ldots \ldots \ldots \ldots \ldots \ldots \ldots \ldots \ldots$ & BS 16469-075 & 13.42 & 0.77 & 50000 & 59 & 145 & 5400 \\
\hline 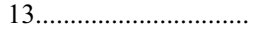 & BS 16920-017 & 13.88 & 0.76 & 50000 & 41 & 100 & 5400 \\
\hline 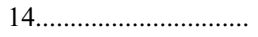 & BS 16928-053 & 13.47 & 0.85 & 50000 & 49 & 120 & 5400 \\
\hline $15 \ldots \ldots \ldots \ldots \ldots \ldots \ldots \ldots \ldots \ldots \ldots \ldots \ldots$ & BS 16929-005 & 13.61 & 0.62 & 50000 & 56 & 137 & 5400 \\
\hline 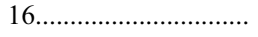 & BS $17583-100$ & 12.37 & 0.51 & 50000 & 79 & 194 & 3600 \\
\hline 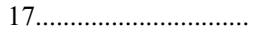 & CS 22169-035 & 12.88 & 0.92 & 50000 & 49 & 120 & 5400 \\
\hline 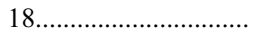 & CS 22183-031 & 13.62 & 0.65 & 50000 & 47 & 115 & 9751 \\
\hline 19 & CS 22892-052 & 13.18 & 0.78 & 90000 & 60 & 147 & 7200 \\
\hline $20 \ldots \ldots \ldots \ldots \ldots \ldots \ldots \ldots$ & CS 22952-015 & 13.27 & 0.78 & 50000 & 59 & 145 & 9000 \\
\hline 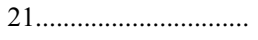 & CS 30306-132 & 12.81 & 0.80 & 50000 & 85 & 208 & 4493 \\
\hline 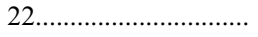 & CS 31082-001 & 11.67 & 0.77 & 50000 & 100 & 122 & 1200 \\
\hline
\end{tabular}

${ }^{\text {a }} \mathrm{S} / \mathrm{N}$ ratio per pixel at $4000 \AA$.

${ }^{b} \mathrm{~S} / \mathrm{N}$ ratio per resolution element at $4000 \AA$.

TABLE 2

Coordinates and Radial Velocity

\begin{tabular}{|c|c|c|c|c|c|}
\hline Number & Star & R.A. (J2000.0) & Decl. (J2000.0) & Observation Date & $V_{r}^{\mathrm{a}}$ \\
\hline 1 & HD 4306 & 004527.2 & -093240 & 2000 Aug 19 & $-69.69 \pm 0.29$ \\
\hline 2 & HD 6268 & 010318.2 & -275250 & 2000 Aug 18 & $39.20 \pm 0.27$ \\
\hline 3....................... & HD 88609 & 101429.0 & +533339 & 2000 Nov 11 & $-37.28 \pm 0.43$ \\
\hline 4 & HD 110184 & 124014.1 & +083138 & 2001 Jan 29 & $138.89 \pm 0.27$ \\
\hline 5 & HD 115444 & 131642.5 & +362253 & $2000 \mathrm{Jul} 4$ & $-27.30 \pm 0.34$ \\
\hline 5 & HD 115444 & & & 2001 Jan 28 & $-27.58 \pm 0.25$ \\
\hline 6 & HD 122563 & 140231.9 & +094110 & $2000 \mathrm{Jul} 4$ & $-27.20 \pm 0.33$ \\
\hline 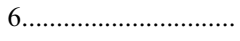 & HD 122563 & & & 2001 Jan 29 & $-26.52 \pm 0.34$ \\
\hline 7........................... & HD 126587 & 142700.4 & -221439 & 2001 Jan 27 & $148.72 \pm 0.72$ \\
\hline 7.......................... & HD 126587 & & & 2001 Jan 31 & $149.10 \pm 0.24$ \\
\hline 8 & HD 140283 & 154303.1 & -105601 & 2000 Jul 4 & $-171.17 \pm 0.29$ \\
\hline 8 & HD 140283 & & & 2000 Aug 17 & $-170.23 \pm 0.19$ \\
\hline 9....................... & HD 186478 & 194514.1 & -172927 & 2000 Aug 20 & $30.52 \pm 0.31$ \\
\hline 10 & BS $16082-129$ & 134711.5 & +285746 & 2001 Jan 30 & $-92.16 \pm 0.30$ \\
\hline $11 \ldots \ldots \ldots \ldots \ldots \ldots \ldots$ & BS $16085-050$ & 123746.7 & +192244 & 2001 Jan 31 & $-75.06 \pm 0.28$ \\
\hline $12 \ldots \ldots \ldots \ldots \ldots \ldots$ & BS $16469-075$ & 101510.1 & +425319 & 2001 Jan 28 & $332.88 \pm 0.74$ \\
\hline $13 \ldots \ldots \ldots \ldots \ldots \ldots \ldots$ & BS $16920-017$ & 120717.1 & +413935 & 2001 Jan 27 & $-206.53 \pm 0.84$ \\
\hline 14 & BS $16928-053$ & 122228.1 & +341124 & 2001 Jan 28 & $-81.00 \pm 0.35$ \\
\hline $15 \ldots \ldots \ldots \ldots \ldots \ldots \ldots$ & BS 16929-005 & 130329.4 & +335106 & 2001 Jan 30 & $-51.29 \pm 0.43$ \\
\hline 16 & BS $17583-100$ & 214227.8 & +264034 & 2000 Aug 19 & $-107.96 \pm 0.32$ \\
\hline $16 \ldots \ldots$ & BS $17583-100$ & & & 2000 Aug 20 & $-108.76 \pm 0.42$ \\
\hline 17 & CS 22169-035 & 041213.9 & -120505 & 2000 Nov 11 & $17.72 \pm 0.73$ \\
\hline 18 & CS 22183-031 & 010904.9 & -044325 & 2000 Nov 10 & $11.67 \pm 0.67$ \\
\hline 18 & CS 22183-031 & & & 2000 Nov 11 & $11.97 \pm 0.56$ \\
\hline 19 & CS 22892-052 & 221701.5 & -163926 & $2001 \mathrm{Jul} 22$ & $12.72 \pm 0.49$ \\
\hline $20 \ldots \ldots \ldots \ldots \ldots \ldots \ldots \ldots \ldots \ldots$ & CS 22952-015 & 233728.6 & -054756 & 2000 Nov 11 & $-20.07 \pm 0.71$ \\
\hline 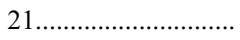 & CS 30306-132 & 151418.6 & +072702 & 2001 Jul 26 & $109.01 \pm 0.31$ \\
\hline 22 & CS 31082-001 & 012931.2 & -160048 & $2001 \mathrm{Jul} 30$ & $138.91 \pm 0.30$ \\
\hline
\end{tabular}

Note.-Units of right ascension are hours, minutes, and seconds, and units of declination are degrees, arcminutes, and arcseconds.

${ }^{a}$ Heliocentric radial velocity $\left(\mathrm{km} \mathrm{s}^{-1}\right)$. 


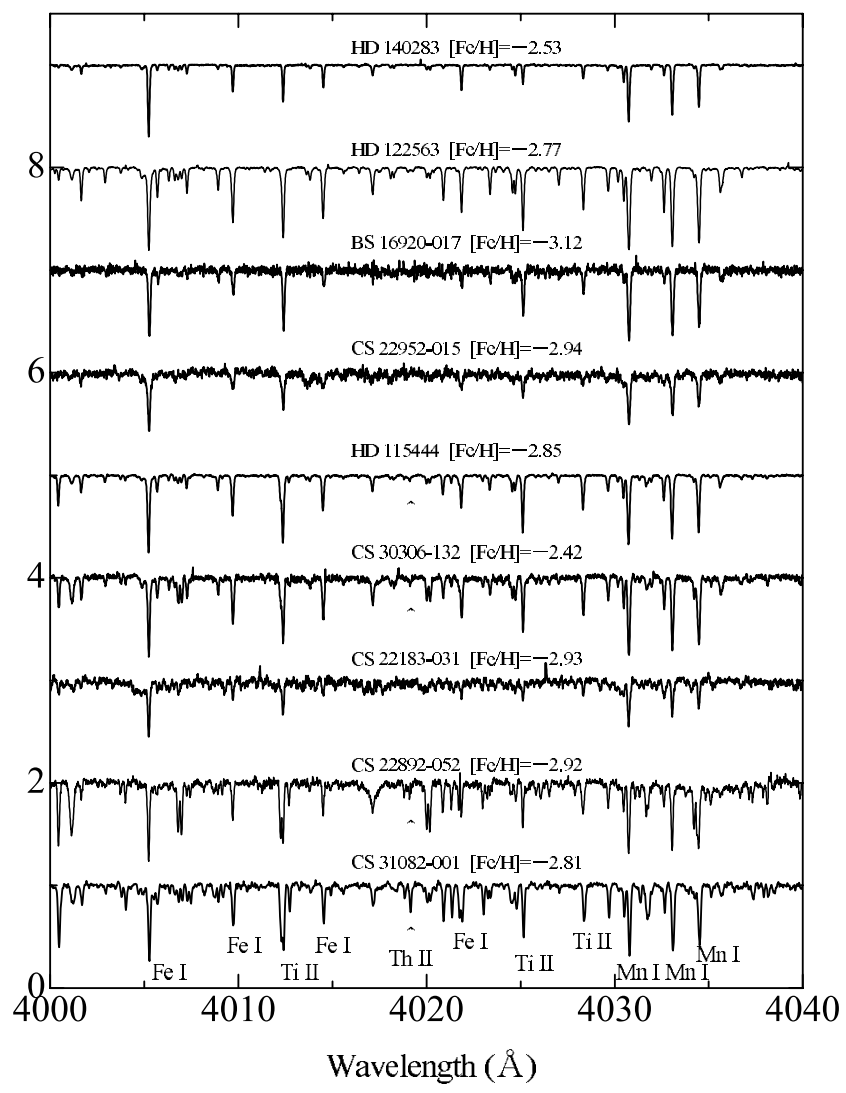

FIG. 1.-Examples of spectra for nine stars in our sample over the wavelength range 4000-4040 $\AA$. The star names and $[\mathrm{Fe} / \mathrm{H}]$ values reported in Paper II are presented in the figure. This spectral region includes the line of Th II at $4019 \AA$ A; detections are indicated with a vertical arrow. The apparent emission lines seen in some of the spectra result from imperfect cosmic-ray cleaning.

the neutron-capture-enhanced, metal-poor stars exhibit abundance patterns associated with $s$-process nucleosynthesis (e.g., Norris, Ryan, \& Beers 1997; Van Eck et al. 2001; Aoki et al. 2002; Lucatello et al. 2003).

These efforts are having a large collective impact on studies on the origin of the neutron-capture elements in the Galaxy (e.g., Ishimaru \& Wanajo 1999; Fields, Truran, \& Cowan 2002; Qian \& Wasserburg 2002), and on the underlying physics and astrophysical sites of the $r$ - and $s$-processes (e.g., Gallino et al. 1998; Wanajo et al. 2002, 2003; Schatz et al. 2002; Truran et al. 2002). Furthermore, detailed studies of the $r$-process-enhanced, very metal-poor stars have provided new, potentially quite powerful, methods for obtaining hard lower limits on the age of the Galaxy and the universe, from the application of cosmo-chronometry based on the observed (present-day) abundance ratios of radioactive nuclei (Th and $\mathrm{U})$, as compared with one another, and with stable elements originating in the $r$-process, (e.g., Eu, Sneden et al. 1996; Westin et al. 2000; Cayrel et al. 2001; Schatz et al. 2002; Wanajo et al. 2002, 2003; Sneden et al. 2003).

In order to develop a more clear understanding of the individual nucleosynthetic processes that were operating in the early Galaxy, further abundance studies are required, based on high-quality spectra, for much larger samples of very metalpoor stars than have been examined to date. We have initiated such a set of investigations with the Subaru Telescope High Dispersion Spectrograph (HDS, Noguchi et al. 2002). In this paper we present observations of 22 very metal-poor stars observed during the commissioning phase of this instrument. In $\S 2$ we discuss the selection of targets and details of the observations that have been carried out. Our spectra cover the wavelength range from 3500 to $5100 \AA$ with high spectral resolution (a resolving power of $R=50,000$ or 90,000) and high signal-to-noise ( $\mathrm{S} / \mathrm{N} \gtrsim 100$ per resolution element). We report the equivalent widths measured for the spectra in $\S 3$, where we also discuss the random errors of our measurements and make comparisons with previous studies of stars in common. Radial velocity measurements for our program stars are presented in $\S 4$, along with a comparison with previous measurements for a number of stars. These data will be used in the detailed abundance analyses that will follow in additional papers of this series.

\section{OBSERVATIONS}

\subsection{Selection of Targets}

The present work is focused primarily on the observed abundance patterns of $r$-process elements in very metal-poor stars. Accordingly, our sample was selected to include stars that fall into one of several categories: (1) very metal-poor stars that were previously known to exhibit extremely large enhancements of their $r$-process elements (CS 22892-052 and CS 31082-001: Sneden et al. 1996; Cayrel et al. 2001); (2) bright metal-poor stars that were studied by previous authors (e.g., McWilliam et al. 1995a; Burris et al. 2000) and shown to be moderately $r$-process-element-rich; and (3) candidate very metal-poor giants discovered in the course of the HK survey of Beers and colleagues (Beers et al. 1992;

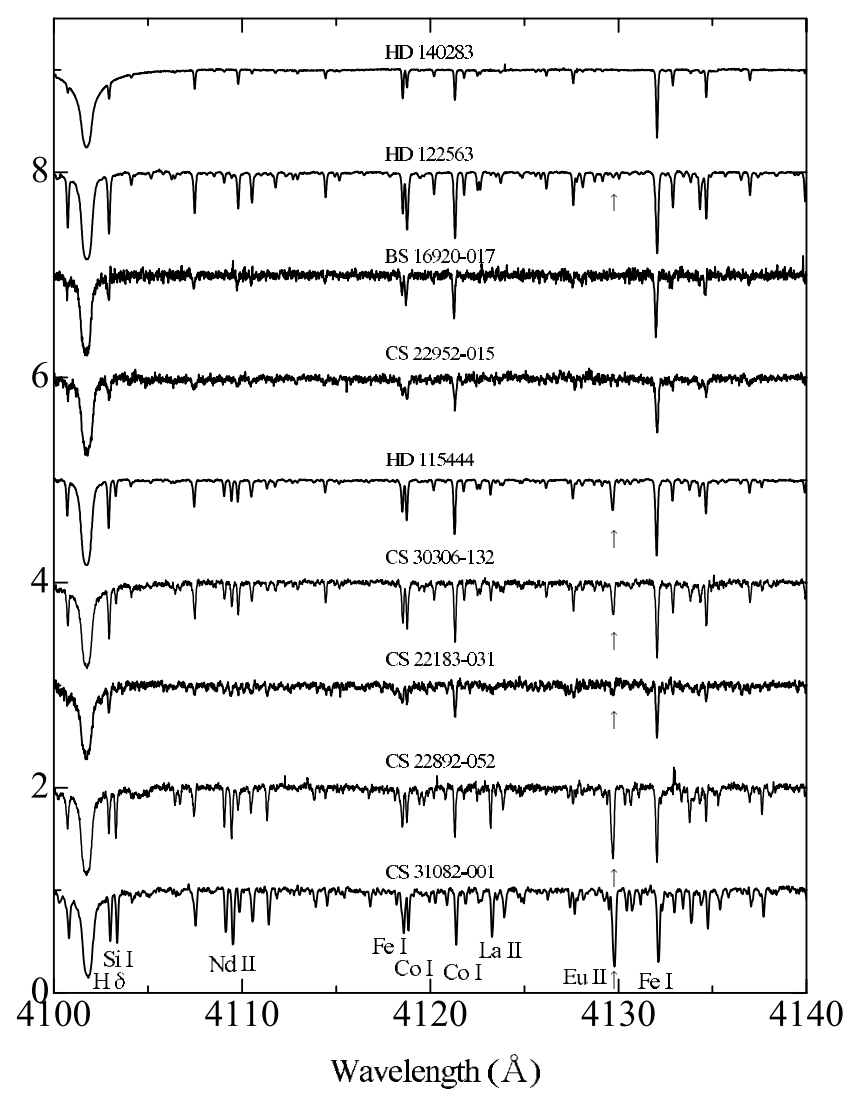

FIG. 2.- Same as Fig. 1, but for the spectral range 4100-4140 A. This region includes lines of $\mathrm{H} \delta$ (from which one may ascertain that the effective temperatures of these stars are quite similar to one another) and the Eu II $4129 \AA$ line; detections are indicated with a vertical arrow. 
TABLE 3

Equivalent Widths for Program Stars

\begin{tabular}{|c|c|c|c|c|c|c|c|c|c|c|c|c|c|c|c|c|c|c|c|c|c|c|c|c|c|}
\hline \multirow{2}{*}{$\begin{array}{l}\text { WAVELENGTH } \\
(\AA)\end{array}$} & \multirow[b]{2}{*}{ SPECIES } & \multirow{2}{*}{$\begin{array}{r}\text { L.E.P. } \\
(\mathrm{eV})\end{array}$} & \multirow[b]{2}{*}{$\log g f$} & \multicolumn{22}{|c|}{ EQUIVALENT WIDTH $(\mathrm{m} \AA)^{\mathrm{a}}$} \\
\hline & & & & 1 & 2 & 3 & 4 & 5 & 6 & 7 & 8 & 9 & 10 & 11 & 12 & 13 & 14 & 15 & 16 & 17 & 18 & 19 & 20 & 21 & 22 \\
\hline $829.35 \ldots \ldots$. & Mg I & 2.71 & -0.480 & 157.6 & 198.5 & 156.4 & & & & & 125.3 & & 131.5 & 158 & 131.2 & 110.3 & 151.6 & 109.4 & 125.2 & 154 & 131.5 & 140.6 & 96.5 & 172.5 & \\
\hline $32.31 \ldots \ldots$ & $M g_{I}$ & 71 & 0.145 & 190.1 & 213.5 & 160.4 & 263.1 & 181.6 & 230 & 174.9 & 153.2 & 231.7 & 143.4 & 177 & 137.9 & 103.6 & 142.5 & 120 & 142.2 & 124.4 & 122.6 & 158.7 & 103.9 & 208.8 & 191 \\
\hline $838.30 \ldots \ldots$ & $\mathrm{Mg}_{\mathrm{I}}$ & .72 & 0.414 & 237.7 & 261.9 & 194.9 & & 205.4 & 262 & 202 & 173.5 & 288.8 & 166.2 & 198 & 163.7 & 137.1 & 171 & 127.9 & 164.7 & 156.6 & 143.7 & 176.2 & 120.5 & 265.3 & \\
\hline $571.10 \ldots \ldots$. & $\mathrm{Mg}_{\mathrm{I}}$ & 0.00 & -5.569 & 45.5 & 77.4 & 76.5 & 132.6 & 51.7 & 85 & 43.8 & 7.7 & 95.7 & 32.2 & 42.2 & 24.1 & 17.5 & 49 & 15.4 & 10.9 & 49.7 & 21.6 & 27.4 & 17.1 & 59.2 & 55.3 \\
\hline $4703.00 \ldots \ldots$ & $\mathrm{Mg}_{\mathrm{I}}$ & 4.35 & -0.377 & $\ldots$ & $\ldots$ & & & 54.6 & 72 & 52.6 & 41 & 90.7 & 45 & 56.5 & & . & $\ldots$ & $\ldots$ & 39.9 & $\ldots$ & 38.2 & 41.5 & 23.7 & 74.3 & 61.9 \\
\hline $5172.70 \ldots \ldots$ & $\mathrm{Mg}_{\mathrm{I}}$ & 2.71 & -0.381 & $\ldots$ & $\ldots$ & & $\ldots$ & $\ldots$ & 209 & 168.4 & & $\ldots$ & 150.4 & 176.8 & 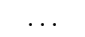 & 131.6 & $\ldots$ & $\ldots$ & $\ldots$ & $\ldots$ & & 154.5 & & 193 & 187.3 \\
\hline $5183.62 \ldots \ldots$. & $\mathrm{Mg}_{\mathrm{I}}$ & 2.72 & -0.158 & $\ldots$ & $\ldots$ & $\ldots$ & $\ldots$ & $\ldots$ & 232 & 187.1 & $\ldots$ & $\ldots$ & 169.6 & 192.4 & $\ldots$ & 149.7 & $\ldots$ & $\ldots$ & $\ldots$ & $\ldots$ & $\ldots$ & 177.4 & & 231.7 & \\
\hline $3961.53 \ldots \ldots .$. & $\mathrm{Al}$ I & 0.01 & -0.336 & 106.9 & 139.5 & 118.1 & 174.3 & 112.4 & 146 & 104.6 & 68.4 & 145.3 & 88 & 105 & 85.5 & 85.1 & 97.5 & 64.2 & 67.1 & 101.6 & 81.4 & 107 & 93.2 & 119.1 & 110.2 \\
\hline $102.94 \ldots \ldots$. & Si I & 1.91 & -3.100 & 63 & 91.4 & 76.6 & 114.5 & 57.4 & 82 & 60.5 & 19.4 & 89 & 46.8 & 73.2 & 40 & 22.9 & 56.1 & 18.2 & 15.5 & 68.3 & 40.1 & 44.6 & 45.6 & 68.5 & 66.9 \\
\hline $226.73 \ldots \ldots$ & $\mathrm{Ca}$ I & 0.00 & 0.240 & $\ldots$ & $\ldots$ & $\ldots$ & $\ldots$ & $\cdots$ & $\ldots$ & $\ldots$ & $\ldots$ & $\ldots$ & $\ldots$ & $\ldots$ & $\ldots$ & & $\ldots$ & $\ldots$ & 134.9 & $\ldots$ & $\ldots$ & Syn & $\ldots$ & $\ldots$ & $\ldots$ \\
\hline $283.01 \ldots \ldots$ & $\mathrm{Ca}_{\mathrm{I}}$ & 1.89 & -0.220 & & & & & 46.5 & 65.6 & 52.2 & 29.6 & 84 & & 39.5 & 35.5 & 12.8 & 44.2 & 30 & 28 & $\ldots$ & 16.5 & & 18.3 & & \\
\hline $318.66 \ldots \ldots$. & $\mathrm{Ca}$ I & 1.90 & -0.210 & 47.4 & 64.1 & 43.9 & 81.4 & 40.9 & 55.4 & 40.6 & 27.2 & 69.9 & 37.2 & 36.8 & 24.9 & 25.2 & 40.1 & 18.8 & 26.7 & $\ldots$ & 19.3 & 32 & 26.8 & 54.4 & 48.7 \\
\hline $4425.44 \ldots \ldots$. & $\mathrm{Ca} \mathrm{I}$ & 1.88 & -0.358 & 42.7 & 55 & 39.9 & 71.6 & 34.4 & 49.1 & 35.7 & 22.2 & 63.5 & 33.9 & 31.4 & 29.2 & 11.2 & 41.8 & 20.7 & 22.4 & $\ldots$ & 19 & 35.2 & & 52 & \\
\hline $454.79 \ldots \ldots$ & $\mathrm{Ca} \mathrm{I}$ & .90 & 0.260 & 70.3 & 95.4 & 69.5 & & 64.9 & 79.9 & 64.6 & 48.5 & 97.1 & 57.9 & 60.7 & 42.6 & 30.8 & 61.7 & 35.1 & 43.9 & $\ldots$ & 49.9 & & & 81 & \\
\hline $4455.89 \ldots \ldots$ & $\mathrm{Ca}$ I & 1.90 & -0.510 & & & 35.4 & & & & & & & 24.2 & $\ldots$ & $\ldots$ & $\ldots$ & $\ldots$ & $\ldots$ & 17.5 & $\ldots$ & & 25 & & & \\
\hline $4400.40 \ldots \ldots .$. & Sc II & 0.60 & -0.540 & 56.2 & 86.6 & 67 & & 58.2 & 78.3 & & 17.5 & 79.6 & 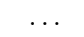 & $\ldots$ & $\ldots$ & $\ldots$ & $\ldots$ & $\ldots$ & 13.5 & $\ldots$ & 30.5 & & 32.3 & & \\
\hline $4415.56 \ldots \ldots .$. & Sc II & 0.59 & -0.670 & 48.9 & 78.1 & 61.7 & 103 & 52.2 & 76 & 47.7 & 14.7 & 80.9 & 37.4 & 51.4 & 29.1 & 29.2 & 41.1 & 3.7 & 13.1 & 46.6 & 22.8 & 41.4 & 23.5 & 50.7 & \\
\hline $5031.02 \ldots \ldots$. & Sc II & 1.36 & -0.400 & 20.3 & 46 & 30.2 & 64.7 & 22.3 & 42 & 18.5 & 4.8 & 47 & 17 & 27.1 & 12.3 & 8.4 & 21.9 & $\ldots$ & & 20.3 & & 15.3 & & 26.2 & 21.8 \\
\hline $3998.64 \ldots \ldots$. & Ti I & 0.05 & -0.056 & 54.8 & $\ldots$ & $\ldots$ & 108.8 & 56 & 70.2 & 52 & 24.2 & 81.5 & & & 28 & $\ldots$ & 53 & 26.2 & 23.3 & $\ldots$ & 32.8 & 37.7 & 33.2 & 59.4 & 59.2 \\
\hline $4533.25 \ldots \ldots$. & Ti I & 0.85 & 0.476 & 38.6 & & & 84.6 & 40.9 & 52.2 & 35. & 14.4 & 63 & 29. & 24. & 20.6 & 30 & 36 & & & & 54.3 & 25.3 & 23 & 47.5 & 104.4 \\
\hline $534.78 \ldots \ldots$. & Ti I & 0.8 & 0.280 & $\ldots$ & $\ldots$ & 36.5 & 71.8 & 31. & 42. & 95 & $\ldots$ & 54.7 & 23 & 17.6 & 16.1 & 19.3 & 30.9 & 14.1 & 10.6 & 36 & $\ldots$ & 21.6 & $\ldots$ & 38.1 & 32 \\
\hline $4535.58 \ldots \ldots$. & Ti I & 0.83 & 0.130 & $\ldots$ & & & & 24.2 & 35.6 & 21.2 & & 49 & 18.8 & 12.4 & $\ldots$ & 14.7 & 29.9 & 10.5 & $\ldots$ & $\ldots$ & & 15.1 & & 30.7 & 27.2 \\
\hline $4981.74 \ldots \ldots$ & Ti I & & .504 & 44.5 & 66.7 & 51 & 97.5 & 47.2 & 61. & 39.8 & 19.1 & 73.3 & 36. & 31 & 25 & 39.2 & $\ldots$ & 16.7 & 19.4 & 36.7 & 22.5 & 31.5 & 15.9 & 54.7 & 48.9 \\
\hline $991.07 \ldots \ldots . .$. & Ti I & & 380 & 40.7 & $\ldots$ & . & & 42.1 & 57. & & 16.4 & 71 & & & & 17.8 & 39.7 & 16 & $\ldots$ & 36.6 & 16.1 & 29.1 & & 49.5 & 44.8 \\
\hline $4999.51 \ldots \ldots .$. & Ti I & 0.83 & 0.250 & 35.8 & 57.3 & 40.4 & 86.1 & 36.3 & 49.1 & 31.6 & 12 & 64.3 & 23.2 & 21.7 & 16.4 & 25.3 & 31 & 13.6 & 15.1 & $\ldots$ & 16.7 & & 12.8 & 42.2 & 47.3 \\
\hline $5039.96 \ldots \ldots$ & Ti I & 0.02 & -1.130 & 17.3 & $\ldots$ & 22.7 & 72.1 & 18.9 & 30.2 & 15.9 & 3.3 & 45.3 & 13.8 & 10.5 & 10.5 & 12.8 & 14.9 & $\ldots$ & $\ldots$ & $\ldots$ & $\ldots$ & 9.7 & $\ldots$ & 20 & 20.6 \\
\hline $5064.66 \ldots \ldots$. & Ti I & 0.05 & -0.991 & 20.5 & $\ldots$ & 29.9 & 78.9 & 22.5 & 35.9 & 18 & 5 & 49.3 & 10.8 & 11.1 & 11.3 & $\ldots$ & 22.3 & $\ldots$ & $\ldots$ & $\ldots$ & $\ldots$ & 12.4 & & 27.9 & \\
\hline $5173.75 \ldots \ldots$ & Ti I & 0.00 & -1.118 & $\ldots$ & $\ldots$ & $\ldots$ & 76.3 & $\ldots$ & 34 & 16.6 & $\ldots$ & $\ldots$ & $\ldots$ & 8.8 & 8.1 & 14.8 & 20.2 & $\ldots$ & $\ldots$ & $\ldots$ & $\ldots$ & $\ldots$ & & 22.1 & . \\
\hline $5192.98 \ldots \ldots .$. & Ti I & 0.02 & 1.006 & $\ldots$ & $\ldots$ & & & & 39.2 & 19.7 & & $\cdots$ & 17.9 & 11.5 & 10 & 15 & 18 & 7 & $\ldots$ & $\ldots$ & $\ldots$ & $\ldots$ & & 28.8 & \\
\hline $4028.35 \ldots \ldots$ & Ti II & 1.89 & 1.000 & 38.4 & & & 82.2 & 42.1 & 52.5 & 35.4 & 13.4 & 70.9 & 27.7 & 25.2 & 21.8 & 26.8 & 33.5 & 12 & 11.3 & $\ldots$ & 16.8 & $\ldots$ & 22.4 & 45.8 & $\ldots$ \\
\hline $4337.93 \ldots \ldots .$. & Ti II & 1.08 & 1.130 & 84.5 & 119 & 80.4 & 145.1 & 92.8 & $\cdots$ & 78 & 40.9 & 110.1 & $\ldots$ & $\ldots$ & $\ldots$ & 70.2 & $\ldots$ & $\ldots$ & 37.4 & 69.6 & 47.5 & $\ldots$ & 50 & 81 & \\
\hline $4394.07 \ldots \ldots .$. & Ti II & 1.22 & -1.590 & 42.3 & 70 & 55 & 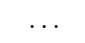 & 48.1 & & & 11.5 & 74 & $\ldots$ & $\ldots$ & $\ldots$ & $\ldots$ & $\ldots$ & $\ldots$ & 12.2 & $\ldots$ & $\ldots$ & $\ldots$ & $\ldots$ & $\ldots$ & $\ldots$ \\
\hline $4395.85 \ldots \ldots$ & $\mathrm{Ti}$ II & 1.24 & -2.170 & 30 & 57.8 & 122.6 & & 36.2 & 46.6 & & 6.7 & 58 & 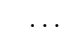 & $\ldots$ & $\cdots$ & $\ldots$ & $\ldots$ & $\ldots$ & $\ldots$ & 103.4 & 64.8 & $\ldots$ & & $\cdots$ & $\ldots$ \\
\hline $4399.78 \ldots \ldots .$. & Ti II & 1.24 & -1.270 & 74.5 & 96.6 & 81.4 & & 75.4 & 89 & & 30.5 & 102.5 & & $\ldots$ & $\ldots$ & $\ldots$ & $\ldots$ & $\ldots$ & 29 & 53.7 & 42.4 & & 35.9 & & \\
\hline $4417.72 \ldots \ldots$ & Ti II & 1.17 & -1.430 & 73.9 & 105 & 88.6 & 129.5 & 81.1 & 95.1 & 70.6 & 32.3 & 104.1 & 62.2 & 60.2 & 52.6 & 63.4 & 69.4 & 29.4 & 28.8 & 67.6 & 34.4 & 65.7 & 41.4 & 86.5 & \\
\hline $4443.81 \ldots \ldots$. & Ti II & 1.08 & -0.700 & 96.8 & 128.2 & 116 & 160.1 & 104 & 119.3 & 95.7 & 60.8 & 126.7 & 85.4 & 90.2 & 76.3 & 87.7 & 100.8 & 55.3 & 57.7 & 91.6 & 61.1 & 84.7 & 73.3 & 100.6 & 101.9 \\
\hline $4450.49 \ldots \ldots$ & Ti II & 1.08 & -1.450 & 64.7 & 96.6 & 75.6 & 124.7 & 71.5 & 87.4 & 63.8 & 23.8 & 92.5 & $\ldots$ & 54.3 & 43.2 & 58.1 & 65.8 & 23.8 & 21.6 & 52.3 & 34.7 & 50.9 & 33.9 & 68.5 & 68.9 \\
\hline $4464.46 \ldots \ldots$. & Ti II & 1.16 & -2.080 & 44.8 & $\ldots$ & $\ldots$ & $\ldots$ & 50.2 & 67 & 42.9 & 11.7 & 76.5 & 35.9 & 32.6 & 22.7 & 38.5 & 45.8 & 18.3 & 12.7 & $\ldots$ & $\ldots$ & 30.5 & $\ldots$ & 50.5 & 47.1 \\
\hline $4468.50 \ldots \ldots$ & Ti II & 1.13 & & 98.4 & 132.5 & 112.8 & 163.8 & 107.1 & 121.4 & 98 & 63.1 & 130. & 83 & 90 & 78 & 89.3 & 101 & 59.6 & 60.3 & 88.6 & 57.8 & 93.3 & 70.9 & 102.4 & \\
\hline $4470.86 \ldots \ldots$ & Ti II & 1.17 & -2.280 & 28.1 & 56.8 & 38.1 & 74.9 & 34.1 & & 26 & 5.4 & 59 & 22 & 18 & 16.6 & 24.5 & 27.3 & $\ldots$ & $\ldots$ & & $\ldots$ & $\ldots$ & $\ldots$ & 34.6 & \\
\hline $4501.28 \ldots \ldots$. & $\mathrm{T}$ & & -0.750 & & $\ldots$ & 109.8 & 156.1 & 101.7 & & & 57.2 & 125.3 & 82.7 & 86 & 70 & 83 & 95 & 55. & 54.2 & 92.5 & 60.7 & 83.2 & 69.9 & 97.9 & 98.5 \\
\hline $4571.98 \ldots \ldots$. & Ti II & & -0.530 & & $\ldots$ & $\ldots$ & 154.6 & 92.6 & 109.9 & 84.5 & 52.6 & 117 & 74 & 80.5 & 64.4 & 76.8 & 87.9 & 44.7 & 49.2 & 85 & 49.1 & 77.2 & 62.1 & 92.2 & 92.3 \\
\hline $4589.95 \ldots \ldots$. & Ti II & 1.24 & -1.790 & 48.4 & $\ldots$ & 61.5 & 102.4 & 54.3 & 71.7 & 46.4 & 13.9 & 78.9 & 40 & 34.3 & 26.2 & 41.6 & 48.5 & 10.2 & 12.5 & 47.9 & 18.4 & 34 & & 52.4 & 55.4 \\
\hline $4865.62 \ldots \ldots .$. & Ti II & 1.12 & -2.610 & 11.3 & 29.7 & 20.9 & 51.8 & 15.1 & 25.2 & 11.5 & 2 & 32.3 & 12 & 9.8 & $\ldots$ & $\ldots$ & 22 & $\ldots$ & $\ldots$ & $\ldots$ & $\ldots$ & 11.1 & $\ldots$ & 14.7 & \\
\hline
\end{tabular}


TABLE 3-Continued

\begin{tabular}{|c|c|c|c|c|c|c|c|c|c|c|c|c|c|c|c|c|c|c|c|c|c|c|c|c|c|}
\hline \multirow{2}{*}{$\begin{array}{c}\text { WAVELENGTH } \\
(\AA)\end{array}$} & \multirow[b]{2}{*}{ SPECIES } & \multirow{2}{*}{$\begin{array}{r}\text { L.E.P. } \\
(\mathrm{eV})\end{array}$} & \multirow[b]{2}{*}{$\log g f$} & \multicolumn{22}{|c|}{ EQUIVALENT WidTh $(\mathrm{m} \AA)^{\mathrm{a}}$} \\
\hline & & & & 1 & 2 & 3 & 4 & 5 & 6 & 7 & 8 & 9 & 10 & 11 & 12 & 13 & 14 & 15 & 16 & 17 & 18 & 19 & 20 & 21 & 22 \\
\hline $5129.16 \ldots \ldots$ & Ti II & 1.89 & -1.390 & & & $\ldots$ & 72.6 & $\ldots$ & 45.1 & 22.8 & $\cdots$ & $\cdots$ & 23.2 & 17.2 & 12.9 & 20.6 & 24.9 & & & & & 18.4 & & 31.6 & \\
\hline $5185.91 \ldots \ldots$. & Ti II & 1.89 & -1.350 & $\ldots$ & $\ldots$ & $\ldots$ & 65.6 & $\ldots$ & 37.1 & 19 & & & 16.5 & 15.4 & 11.1 & 17.9 & 22.6 & & $\ldots$ & $\ldots$ & $\ldots$ & 11.8 & $\ldots$ & 27.1 & 22 \\
\hline $5188.70 \ldots \ldots$ & Ti II & 1.58 & -1.210 & $\ldots$ & $\ldots$ & $\ldots$ & $\ldots$ & $\ldots$ & 90 & 59.8 & $\ldots$ & $\ldots$ & $\ldots$ & $\ldots$ & 34 & 49 & $\ldots$ & 19.2 & $\ldots$ & $\ldots$ & $\ldots$ & $\ldots$ & $\ldots$ & $\ldots$ & $\ldots$ \\
\hline $4379.24 \ldots \ldots .$. & V I & 0.30 & 0.565 & 14.2 & 28.9 & $\ldots$ & $\ldots$ & 14 & 28 & $\ldots$ & 4.9 & 38.6 & $\ldots$ & $\ldots$ & $\ldots$ & $\ldots$ & $\ldots$ & $\ldots$ & $\ldots$ & $\ldots$ & $\ldots$ & $\ldots$ & 11.6 & & \\
\hline $4389.99 \ldots \ldots .$. & V I & 0.28 & 0.235 & $\ldots$ & $\ldots$ & $\ldots$ & $\ldots$ & 8.7 & 21.7 & $\ldots$ & $\ldots$ & $\ldots$ & $\ldots$ & $\ldots$ & $\ldots$ & $\ldots$ & $\ldots$ & $\ldots$ & $\ldots$ & $\ldots$ & $\ldots$ & $\ldots$ & $\ldots$ & . & $\ldots$ \\
\hline 3951.96....... & $\mathrm{V}_{\mathrm{II}}$ & 1.48 & -0.784 & 17.7 & 44.6 & 34.9 & $\ldots$ & 21.6 & 35.4 & 17.4 & 5.2 & 42.5 & 36.1 & 18.2 & 11.6 & . & 16.2 & $\ldots$ & 6.2 & $\ldots$ & 17.1 & 15.7 & $\ldots$ & 23.3 & 29.4 \\
\hline $4005.71 \ldots \ldots$. & V II & 1.82 & -0.522 & 18.1 & 46 & 38.6 & $\ldots$ & 19.3 & 36.3 & 18.4 & 5.2 & 43.5 & 16.9 & 17.6 & 7.1 & 17.6 & 21.1 & $\ldots$ & . & $\cdots$ & $\ldots$ & $\ldots$ & $\ldots$ & 23.2 & 33.5 \\
\hline $4254.35 \ldots \ldots$. & $\mathrm{Cr} \mathrm{I}$ & 0.00 & -0.114 & $\ldots$ & & $\ldots$ & $\ldots$ & & 113.7 & 88 & 66.3 & & 84.2 & 83.7 & 70.7 & 82.9 & 95.6 & 52.4 & 60.1 & & & 87.4 & & 96.8 & 95.7 \\
\hline $4274.81 \ldots \ldots .$. & $\mathrm{Cr}$ I & 0.00 & -0.231 & & & $\ldots$ & $\ldots$ & $\ldots$ & 112.8 & 88.1 & 63.5 & & 82.2 & 79.4 & 69.1 & 79.6 & 92.5 & 52.9 & 53.6 & $\ldots$ & 51.3 & $\ldots$ & $\ldots$ & & 96.7 \\
\hline $4289.73 \ldots \ldots .$. & $\mathrm{Cr} \mathrm{I}$ & 0.00 & -0.361 & 85.9 & 109.8 & 92.1 & 135.1 & 86 & 109.1 & 82 & 57.2 & & 76.8 & 72.6 & 67.2 & 72.9 & 88.3 & 47.6 & 49.6 & 88.1 & 53.1 & $\ldots$ & 66.4 & 96.1 & $\ldots$ \\
\hline $4554.99 \ldots \ldots .$. & $\mathrm{Cr}_{\text {II }}$ & 4.07 & -1.380 & & & $\ldots$ & $\ldots$ & 2.8 & 4.8 & 4.8 & 1.5 & & $\ldots$ & & & & & $\ldots$ & $\ldots$ & $\ldots$ & $\ldots$ & & $\ldots$ & & $\ldots$ \\
\hline $4558.65 \ldots \ldots .$. & $\mathrm{Cr}$ II & 4.07 & -0.660 & 10.2 & 30.2 & 13.4 & $\ldots$ & 11.1 & 20.7 & 20.7 & 8.7 & 28.2 & 9.8 & 11.2 & & 8.9 & 12.2 & $\ldots$ & $\ldots$ & $\ldots$ & $\ldots$ & $\ldots$ & $\ldots$ & 17.9 & $\ldots$ \\
\hline $4588.20 \ldots \ldots$ & $\mathrm{Cr}$ II & 4.07 & -0.630 & 8.4 & 19.8 & 6.8 & 23.5 & 7.1 & 13.3 & 13.3 & 5 & 18.6 & 5.2 & 5 & & 4.1 & 7.9 & $\ldots$ & $\ldots$ & $\ldots$ & $\ldots$ & $\ldots$ & $\ldots$ & 9 & $\ldots$ \\
\hline $4030.76 \ldots \ldots$ & Mn I & 0.00 & -0.470 & 91 & 138.2 & 105.5 & $\ldots$ & 97.2 & 140.8 & 97.2 & 66.8 & 138.8 & 84.8 & 106.1 & 75.5 & 98.7 & 97.1 & 39.5 & 50.4 & 130.3 & 65.1 & 93.6 & 91.6 & 109.8 & 109 \\
\hline 4033.07........ & Mn I & 0.00 & -0.618 & 77.4 & 124.4 & 95.8 & 179.3 & 83.6 & 125.2 & 83.6 & 55.2 & 124.9 & 75.1 & 91.6 & 61.3 & 86.7 & 91.9 & 35.6 & 38.9 & 112.2 & 46.3 & 92.2 & 73.2 & 97.8 & 96.2 \\
\hline $4034.49 \ldots \ldots$. & Mn I & 0.00 & -0.811 & 79.1 & 105.8 & 89.3 & 164.8 & 75.2 & 119.8 & 75.2 & 45.9 & 121.3 & 77.1 & 80.9 & 57.7 & 80.8 & 86.7 & $\ldots$ & 33 & $\ldots$ & 36.4 & $\ldots$ & 57.4 & 108.1 & 91.8 \\
\hline $4041.37 \ldots \ldots$. & Mn I & 2.11 & 0.285 & 16.6 & 45.7 & 37.2 & 67.7 & 15.7 & 43.9 & 15.7 & 13.3 & 47.1 & 23.2 & 26.6 & 7.7 & 31.8 & 26.6 & $\ldots$ & $\ldots$ & $\ldots$ & $\ldots$ & 18.7 & $\ldots$ & 30.8 & 19.8 \\
\hline $4754.04 \ldots \ldots$. & Mn I & 2.28 & -0.086 & 6.3 & 18.1 & 11.6 & 40.6 & 5. & 19.9 & 5.9 & 5.2 & 21 & 6.5 & 9.7 & . & 13.5 & 13.2 & $\ldots$ & $\ldots$ & $\ldots$ & $\ldots$ & 5.5 & $\ldots$ & 12.3 & 7.3 \\
\hline $4823.51 \ldots \ldots .$. & Mn I & 2.32 & 0.144 & 8.8 & 26.2 & 12.8 & $\ldots$ & 8.6 & 25.7 & 8.6 & 7.3 & 28.4 & 11.3 & 14.9 & & 13.3 & 14.3 & & 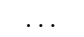 & $\ldots$ & $\ldots$ & $\ldots$ & $\ldots$ & 20.1 & \\
\hline $3763.80 \ldots \ldots .$. & $\mathrm{Fe} \mathrm{I}$ & 0.99 & -0.221 & 139 & 209.9 & $\ldots$ & $\ldots$ & 152.4 & 171.7 & 138.7 & 107.8 & 217.1 & 113.9 & 131.3 & 116.6 & 98 & 137.2 & $\ldots$ & 103 & $\ldots$ & 102.5 & 134.5 & 53.9 & 155 & 140.4 \\
\hline $3767.20 \ldots \ldots .$. & $\mathrm{Fe}$ I & 1.01 & -0.382 & 125.9 & 175.7 & $\ldots$ & & 137.6 & 151.7 & 127.3 & 96.9 & 180.3 & 106.1 & 120.7 & 110.8 & 90.8 & 128.9 & 83.8 & 86.4 & & 84.4 & 127.6 & 109.2 & 137.8 & \\
\hline $3787.89 \ldots \ldots$ & $\mathrm{Fe} \mathrm{I}$ & 1.01 & -0.838 & 112 & 148.2 & 116 & 222.8 & 131.1 & 129.7 & 110.3 & 84.5 & 145.9 & 89.4 & 108.4 & 100.5 & 85.3 & 115.7 & 72 & 80.9 & 130.3 & 63.7 & 109 & 51.2 & 114.8 & 109 \\
\hline $3805.35 \ldots \ldots$ & $\mathrm{Fe}_{\mathrm{I}}$ & 3.30 & 0.313 & 46.8 & 78.8 & $\ldots$ & 81.6 & 58.1 & 59.3 & 48.9 & 40.4 & 76.9 & 52.1 & 42.3 & 42.7 & & 47.8 & 19.2 & 34.3 & $\ldots$ & 31.2 & 65.3 & 47.4 & 63.6 & \\
\hline $3815.85 \ldots \ldots .$. & $\mathrm{Fe} \mathrm{I}$ & 1.49 & 0.237 & 137.2 & 191.1 & $\ldots$ & $\ldots$ & 150.9 & 165.6 & 138.1 & 114.6 & 195.1 & 119.6 & 131.5 & 121.5 & 96.9 & 138.2 & 91.8 & 109 & $\ldots$ & 111.1 & 138.7 & 102.5 & 156.6 & 164 \\
\hline $3820.44 \ldots \ldots .$. & $\mathrm{Fe} \mathrm{I}$ & 0.86 & 0.157 & 188.2 & 287.4 & 252.6 & $\ldots$ & 210.3 & 279.7 & 193.3 & 148.5 & 331.7 & 168.2 & 172.2 & 159.9 & 149.9 & 210.2 & 122 & 134.8 & $\ldots$ & 143.3 & 183.2 & 149.6 & 234 & 210 \\
\hline $3825.89 \ldots \ldots$. & $\mathrm{Fe} \mathrm{I}$ & 0.92 & -0.024 & 165.1 & 234.8 & 198.3 & $\ldots$ & 182.3 & 222.2 & 165.8 & 129.4 & 272.9 & 151.6 & 152.4 & 134.5 & 131 & 180 & 107 & 119 & $\ldots$ & 125.5 & 171.2 & 135.5 & 193.3 & \\
\hline $3827.83 \ldots \ldots$ & $\mathrm{Fe} \mathrm{I}$ & 1.56 & 0.094 & 124.3 & 177.5 & 117 & $\ldots$ & 136 & 159 & 124.5 & 102 & 185.9 & 112.5 & 122.1 & 97.8 & 107.9 & 137.8 & 84.4 & & $\ldots$ & 89.7 & 127.6 & 101.5 & 140 & \\
\hline $3840.45 \ldots \ldots$ & $\mathrm{Fe}_{\mathrm{I}}$ & 0.99 & -0.497 & 123.9 & 171.5 & 138.8 & $\ldots$ & 136.6 & 154.4 & 126.7 & 96.1 & 182.8 & 109.9 & 121.7 & 111.7 & 92.1 & 130 & 82.2 & 91.7 & 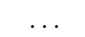 & 88.9 & 121.1 & 109.8 & 139 & 129.4 \\
\hline 3849.98........ & $\mathrm{Fe} \mathrm{I}$ & 1.01 & -0.863 & 110.6 & 149.7 & $\ldots$ & $\ldots$ & 125.2 & 142.2 & 111.9 & 87.3 & 156.4 & 103.7 & 111.7 & 101.2 & 99.5 & 124.7 & 82.3 & 80.3 & 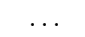 & 78.6 & 117.3 & 105.2 & 119.1 & 119.9 \\
\hline $3856.38 \ldots \ldots .$. & $\mathrm{Fe} \mathrm{I}$ & 0.05 & -1.280 & 137.6 & 180.7 & 160.6 & $\ldots$ & 155.8 & 173.6 & 140.3 & 106.1 & 189.3 & 117.6 & 139.6 & 117.5 & 123.1 & 148.7 & 96 & 98 & 155.9 & 101.5 & 138.1 & 138.9 & 146.4 & 149 \\
\hline $3859.92 \ldots \ldots .$. & $\mathrm{Fe} \mathrm{I}$ & 0.00 & -0.698 & 184 & 234.7 & 203.7 & $\ldots$ & 190.7 & 238.2 & 185.3 & 137.5 & 292.4 & 151.9 & 168.5 & 156 & 142.5 & 195.6 & 116.1 & 126.6 & 182.3 & 121 & 179 & 144.1 & 215.2 & 191.1 \\
\hline $3865.53 \ldots \ldots$. & $\mathrm{Fe}_{\mathrm{I}}$ & 1.01 & -0.950 & 111.2 & 144.8 & 115.9 & 194.6 & 119.6 & 136.1 & 111.7 & 84.1 & 148.2 & 100.7 & 110.3 & 100.9 & 90.4 & 117.7 & 72.5 & 78.7 & 128.6 & 82.2 & 111.1 & 102 & 115.8 & 118.7 \\
\hline $3886.29 \ldots \ldots$. & $\mathrm{Fe} \mathrm{I}$ & 0.05 & -1.055 & 115.4 & 208.5 & 161.7 & $\ldots$ & 165.3 & 184.7 & 156 & 109 & 221.3 & 127.6 & 141.4 & 144.7 & 125.8 & 150.4 & 103.5 & 105.5 & 174.1 & 104.8 & 193.4 & 132.8 & 179.6 & 173.3 \\
\hline $3899.72 \ldots \ldots .$. & $\mathrm{Fe} \mathrm{I}$ & 09 & -1.515 & 127.7 & 165.9 & 150.1 & $\ldots$ & 140.5 & 162.5 & 129.6 & 96.7 & 168.2 & 121 & 133.8 & 122.7 & 118 & 140 & 93 & 93.3 & 158.4 & 91 & 130.7 & 133.3 & 132.7 & 139.3 \\
\hline $3902.96 \ldots \ldots .$. & $\mathrm{Fe} \mathrm{I}$ & 1 & -0.442 & 103.8 & 138.9 & 118.5 & $\ldots$ & 112.1 & 131.4 & 105.5 & 82.2 & 123.5 & 98.2 & 103.5 & 89 & 92.4 & 111.8 & 68.8 & $\cdots$ & $\ldots$ & 76.3 & 105.9 & 11.3 & 111.3 & 108.1 \\
\hline $3920.27 \ldots \ldots .$. & $\mathrm{Fe} \mathrm{I}$ & 0.12 & -1.734 & 121.8 & 161.7 & 142.5 & $\ldots$ & 137 & 157.7 & 123.1 & 89.3 & 161.7 & 119.8 & 122.6 & 115.9 & 114.1 & 137.1 & 88.4 & 84.6 & $\cdots$ & 99.5 & 125.8 & 118.2 & 121.8 & 126.2 \\
\hline $3922.92 \ldots \ldots$. & $\mathrm{Fe} \mathrm{I}$ & 0.05 & -1.626 & 128.9 & 171.7 & 148.1 & 253.8 & 142.5 & 168.4 & 132 & 96.1 & 173.5 & 124.2 & 132.4 & 120.6 & 114.2 & 148.2 & 95.1 & 91.4 & 164.3 & 93.6 & 128 & 130.3 & 135.7 & 133.5 \\
\hline $3949.96 \ldots \ldots .$. & $\mathrm{Fe} \mathrm{I}$ & 2.18 & -1.251 & 45.5 & 68.5 & 61 & 95 & 47.7 & 66.9 & 44.9 & 28.3 & 73.8 & 37.2 & 36.1 & 30.8 & 26.8 & 48.1 & 16.5 & 23.2 & 59.4 & 36.9 & 42.9 & 30.1 & 55.9 & 50.7 \\
\hline $4005.25 \ldots \ldots$. & $\mathrm{Fe}_{\mathrm{I}}$ & 1.56 & -0.583 & 101.5 & 142.7 & 110.1 & $\ldots$ & 110.4 & 131.7 & 103.1 & 78.8 & 142.4 & 98.8 & 99.4 & 87 & 85 & 112 & 67.2 & 71.5 & 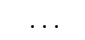 & 81.8 & 100.4 & 102.5 & 104.7 & 107.5 \\
\hline $4063.61 \ldots \ldots$. & $\mathrm{Fe} \mathrm{I}$ & 1.56 & 0.062 & 128.4 & 186.3 & 147.7 & $\ldots$ & 139.4 & 166.3 & 129.6 & 106.7 & 179.9 & 118.6 & 127.5 & 115.9 & 110.3 & 137.6 & 88.3 & 96.5 & $\ldots$ & 110.1 & 135.7 & 123.7 & 144.9 & \\
\hline $4071.75 \ldots \ldots$ & $\mathrm{Fe} \mathrm{I}$ & 1.61 & -0.008 & 120.6 & 168.7 & 150 & $\ldots$ & 130.1 & 153.7 & 120.8 & $\ldots$ & 171.2 & 109.6 & 117.3 & 107.4 & 72.6 & 115.7 & 80.9 & 91.7 & $\ldots$ & 76.9 & 117.3 & 113.8 & 130.9 & 128.7 \\
\hline $4076.64 \ldots \ldots$. & $\mathrm{Fe} \mathrm{I}$ & 3.21 & -0.528 & 26.7 & $\ldots$ & 25.8 & $\ldots$ & 29.4 & 45.1 & 27.2 & 18.5 & 59.8 & $\ldots$ & 21.9 & & $\ldots$ & $\ldots$ & 16 & 15.5 & $\ldots$ & $\ldots$ & 34.6 & $\ldots$ & 39.4 & \\
\hline $4114.45 \ldots \ldots .$. & $\mathrm{Fe} \mathrm{I}$ & 2.83 & -1.303 & 12.8 & 33.3 & 16.8 & 52.8 & 14.3 & 28.8 & 13 & 8.7 & 38.9 & 19.6 & 10.7 & & & 16.7 & $\ldots$ & $\ldots$ & $\ldots$ & $\ldots$ & 10.2 & 17.4 & 18.8 & 16.6 \\
\hline $4132.91 \ldots \ldots$ & $\mathrm{Fe} \mathrm{I}$ & 2.85 & -1.005 & 23.1 & 47.2 & 119.5 & 184.4 & 22.7 & 42.2 & 102.7 & 15.3 & 143.8 & 90.2 & & 87.2 & & 24.7 & & 17.3 & & 67.3 & 101.6 & 95.4 & 33.8 & \\
\hline $4134.69 \ldots \ldots$. & $\mathrm{Fe} \mathrm{I}$ & 2.83 & -0.649 & 36.3 & 66 & 48 & $\ldots$ & 43.8 & 58.3 & 38 & 28.2 & 68.6 & 37.1 & & 21.4 & & 37.8 & 15.7 & 21.3 & $\ldots$ & 18.5 & 34.6 & 33.8 & 53.6 & 48.9 \\
\hline $4143.88 \ldots \ldots$ & $\mathrm{Fe}_{\mathrm{I}}$ & 1.56 & -0.511 & 104 & 134.7 & 123.9 & & 113.2 & 134.5 & 104.8 & 83.7 & 135.3 & 100.1 & 105.5 & 94.4 & 102.3 & 117.5 & 75.9 & 81.9 & & 72.5 & 104.2 & 101.3 & 111.1 & 50.4 \\
\hline
\end{tabular}


TABLE 3-Continued

\begin{tabular}{|c|c|c|c|c|c|c|c|c|c|c|c|c|c|c|c|c|c|c|c|c|c|c|c|c|c|}
\hline \multirow{2}{*}{$\begin{array}{c}\text { WAVELENGTH } \\
(\AA)\end{array}$} & \multirow[b]{2}{*}{ SPECIES } & \multirow{2}{*}{$\begin{array}{r}\text { L.E.P. } \\
(\mathrm{eV})\end{array}$} & \multirow[b]{2}{*}{$\log g f$} & \multicolumn{22}{|c|}{ EquiVALENT WIDTH $(\mathrm{m} \AA)^{\mathrm{a}}$} \\
\hline & & & & 1 & 2 & 3 & 4 & 5 & 6 & 7 & 8 & 9 & 10 & 11 & 12 & 13 & 14 & 15 & 16 & 17 & 18 & 19 & 20 & 21 & 22 \\
\hline $4147.68 \ldots \ldots$. & $\mathrm{Fe}_{\mathrm{I}}$ & 1.49 & -2.071 & 47.5 & 82.1 & 64 & 112.5 & 57.5 & 78.7 & 49.5 & 22.5 & 85.5 & 46.6 & 40 & 35.3 & $\ldots$ & 59.9 & 18 & 15.2 & $\ldots$ & 15.7 & 49.2 & 35.6 & 58.7 & 57.9 \\
\hline $4154.51 \ldots \ldots$. & $\mathrm{Fe}_{\mathrm{I}}$ & 2.83 & -0.688 & 38.1 & 49.4 & 36.9 & $\ldots$ & 32.1 & 52.4 & 23 & 21.3 & 64.7 & 27.2 & 24 & & $\ldots$ & 36.2 & $\ldots$ & 21.5 & $\ldots$ & $\ldots$ & & & 51.9 & \\
\hline $4156.81 \ldots \ldots$. & $\mathrm{Fe}_{\mathrm{I}}$ & 2.83 & -0.808 & 39.6 & 63 & 37.5 & & 34.5 & 59.3 & 33.6 & 22.5 & 69.2 & 29 & 27.1 & 17.9 & $\ldots$ & 37 & $\ldots$ & & & & 28.5 & & 46.1 & \\
\hline $4157.79 \ldots \ldots$ & $\mathrm{Fe}_{\mathrm{I}}$ & 3.42 & -0.403 & 27.8 & 38.8 & 24 & 58.2 & 22.2 & $\ldots$ & 22.7 & 13.9 & 57.7 & 19.7 & 15.6 & 17 & $\ldots$ & $\ldots$ & $\ldots$ & $\ldots$ & $\ldots$ & $\ldots$ & 29.2 & & 35.6 & $\ldots$ \\
\hline $4174.92 \ldots \ldots$ & $\mathrm{Fe}_{\mathrm{I}}$ & 0.92 & -2.938 & 41.9 & 76.2 & 70.2 & 130.4 & 58.1 & 78.6 & 44.7 & 14.3 & 84.6 & $\ldots$ & 31.9 & 25.7 & 30.9 & $\ldots$ & $\ldots$ & $\ldots$ & 66.9 & $\ldots$ & 45 & 33.8 & 57.3 & 53.8 \\
\hline $4175.64 \ldots \ldots$. & $\mathrm{Fe}_{\mathrm{I}}$ & 2.85 & -0.827 & 30.1 & 66.2 & 41.8 & 78.9 & 42.4 & 52.5 & 31.1 & 20.9 & 64.5 & $\ldots$ & 20.9 & $\ldots$ & $\ldots$ & $\ldots$ & 14.8 & 17.7 & $\ldots$ & $\ldots$ & 41.3 & 20.1 & 45.6 & \\
\hline $4181.76 \ldots \ldots$. & $\mathrm{Fe}$ I & 2.83 & -0.371 & 53.2 & 79.5 & 57.2 & $\ldots$ & $\ldots$ & 69.8 & 50.5 & 38.7 & 84.2 & 47.1 & 41.2 & 38.8 & $\ldots$ & 53.5 & 25.2 & $\ldots$ & $\ldots$ & $\ldots$ & 56.5 & $\ldots$ & 59.6 & 57.2 \\
\hline $4182.39 \ldots \ldots$ & $\mathrm{Fe}_{\mathrm{I}}$ & 3.02 & -1.180 & 8.6 & 25.9 & 10.2 & 40.9 & $\ldots$ & 18.9 & 10.2 & 5.7 & 27.5 & $\ldots$ & $\ldots$ & $\ldots$ & $\ldots$ & $\ldots$ & $\ldots$ & $\ldots$ & $\ldots$ & $\ldots$ & $\ldots$ & $\ldots$ & 15.6 & $\ldots$ \\
\hline $4187.05 \ldots \ldots$. & $\mathrm{Fe}$ I & 2.45 & -0.514 & 64.7 & 91.4 & 77.4 & 123.5 & 82.7 & & 64 & 51.7 & 94.1 & 53.6 & 55.9 & 51.5 & 44.4 & 74.2 & 31.8 & 38.7 & 86.7 & 35.3 & 61.8 & 55.4 & 74.1 & 71.8 \\
\hline $4187.81 \ldots \ldots$. & $\mathrm{Fe}$ I & 2.42 & -0.510 & 66.5 & 97.9 & 77.1 & & 70.4 & & 69 & 57.8 & 103.1 & 63.7 & 59.6 & 55 & 54.6 & 73.5 & 38.3 & 40.6 & & 31.7 & 69.6 & 57.8 & 80.6 & 75.7 \\
\hline $4191.44 \ldots \ldots$. & $\mathrm{Fe} \mathrm{I}$ & 2.47 & -0.666 & 54.7 & 90.3 & 61.6 & $\ldots$ & 59.2 & 79.5 & 56.8 & & 84.8 & 51.7 & 50.8 & 41.3 & 41.8 & 62.1 & 22.7 & 32.9 & & & 55.3 & & 69.2 & 68.8 \\
\hline $4195.34 \ldots \ldots$. & $\mathrm{Fe}_{\mathrm{I}}$ & 3.33 & -0.492 & 30.5 & 39.3 & 25.5 & 73.7 & 30.3 & 43 & 27.7 & 16.6 & 56.4 & 28.7 & 17.3 & 17.3 & & 28.3 & & 16 & & & & & 41.4 & 32.3 \\
\hline $4199.10 \ldots \ldots$ & $\mathrm{Fe}_{\mathrm{I}}$ & 3.05 & 0.156 & 61 & 85.3 & 74.3 & $\ldots$ & 63.7 & 80.9 & 60.4 & 48.3 & 90.2 & 56.2 & 53.6 & 47.5 & 38 & 63.7 & 32.2 & 43.1 & 78.1 & 28.3 & 72.7 & 51.3 & 70.6 & 74.2 \\
\hline $4202.04 \ldots \ldots$ & $\mathrm{Fe}_{\mathrm{I}}$ & 1.49 & -0.689 & 102.3 & 137.5 & 113.1 & $\ldots$ & 110 & 132.1 & 104.6 & 80.2 & 126.2 & 99.4 & 101.3 & 90.3 & 94.2 & 112.2 & 72.4 & 76.3 & 140.3 & 73.7 & 100.9 & & 106.7 & 115 \\
\hline $4222.22 \ldots \ldots$ & $\mathrm{Fe}_{\mathrm{I}}$ & 2.45 & -0.914 & 45.6 & 74.4 & $\ldots$ & 101.9 & 48.6 & 73.4 & 47.3 & & 78.6 & 46.6 & 40.3 & 32.7 & 29.9 & 58.1 & 20.1 & $\ldots$ & 66.2 & 25.9 & 40.8 & 30.1 & 59.2 & 60.1 \\
\hline $4227.44 \ldots \ldots$. & $\mathrm{Fe} \mathrm{I}$ & 3.33 & 0.266 & $\ldots$ & 93.7 & 88.3 & $\ldots$ & 64 & 96 & 59.6 & 42.8 & $\ldots$ & 53.8 & 49.4 & 39.7 & 40.2 & 67.7 & 28.8 & $\ldots$ & $\ldots$ & $\ldots$ & 54 & 38.4 & 76.9 & 68.8 \\
\hline $4233.61 \ldots \ldots$. & $\mathrm{Fe}_{\mathrm{I}}$ & 2.48 & -0.579 & $\ldots$ & $\ldots$ & $\ldots$ & 119.7 & & 85.9 & 60.5 & 43.5 & & 57.2 & 54.6 & 45.3 & 58.9 & 66.7 & 29.5 & $\ldots$ & $\ldots$ & & 58.3 & & 73.4 & 68.3 \\
\hline $4250.13 \ldots \ldots$ & $\mathrm{Fe}_{\mathrm{I}}$ & 2.47 & -0.380 & $\ldots$ & $\ldots$ & $\ldots$ & 123.3 & $\ldots$ & 96.3 & 70.1 & 53.8 & $\ldots$ & 61.7 & 62.3 & 53.8 & 46.7 & 74.7 & 34.5 & $\ldots$ & $\ldots$ & $\ldots$ & 63.8 & & 78.2 & 83.5 \\
\hline $4250.80 \ldots \ldots$ & $\mathrm{Fe}_{\mathrm{I}}$ & 1.56 & -0.713 & $\ldots$ & $\ldots$ & $\ldots$ & 166.2 & $\ldots$ & 129.9 & 100.6 & 77.8 & $\ldots$ & 98.8 & 98.1 & 89.8 & 87.6 & 108.5 & 62 & & $\ldots$ & & 96.1 & & 105 & 109.8 \\
\hline $4260.49 \ldots \ldots$ & $\mathrm{Fe}_{\mathrm{I}}$ & 2.40 & 0.077 & $\ldots$ & $\ldots$ & $\ldots$ & $\ldots$ & $\ldots$ & 122.6 & 95 & 77.9 & & 88.2 & 91 & 79.1 & 79.9 & 98.3 & 55.7 & & $\ldots$ & & 88.5 & & 100.7 & 99.4 \\
\hline $4271.16 \ldots \ldots$ & $\mathrm{Fe}_{\mathrm{I}}$ & 2.45 & -0.337 & $\ldots$ & $\ldots$ & $\ldots$ & $\ldots$ & $\ldots$ & 106.9 & 82.8 & 55.4 & & $\ldots$ & 70.5 & 64.8 & 118.9 & 84.3 & 54.8 & $\ldots$ & $\ldots$ & & $\ldots$ & & 101.2 & $\ldots$ \\
\hline $4325.77 \ldots \ldots .$. & $\mathrm{Fe}_{\mathrm{I}}$ & 1.61 & 0.006 & 125.6 & 173.9 & 150.7 & $\ldots$ & 134 & 163.3 & 130.9 & 101.3 & 180.7 & $\ldots$ & 124 & $\ldots$ & 123.8 & 140.8 & 89 & 97.4 & $\ldots$ & 97.5 & & 123.7 & 146.3 & $\ldots$ \\
\hline $4337.06 \ldots \ldots$ & $\mathrm{Fe}_{\mathrm{I}}$ & 1.56 & -1.704 & 62.1 & 94.1 & 74.1 & 136.2 & 69.4 & 88.9 & 65.5 & 32.8 & 93.5 & $\ldots$ & $\ldots$ & 49.6 & $\ldots$ & & $\ldots$ & 32.7 & & 40.1 & & 44.6 & 76.3 & $\ldots$ \\
\hline $4383.56 \ldots \ldots$ & $\mathrm{Fe}_{\mathrm{I}}$ & 1.49 & 0.208 & 143.6 & 195.1 & 175.6 & $\ldots$ & 151.5 & 188.2 & & 122.4 & 199.3 & $\ldots$ & $\ldots$ & $\ldots$ & $\ldots$ & $\ldots$ & $\ldots$ & 111.8 & 178.7 & & & 136.9 & $\ldots$ & $\ldots$ \\
\hline $4404.76 \ldots \ldots$. & $\mathrm{Fe}_{\mathrm{I}}$ & 1.56 & -0.147 & 123.3 & 168.1 & 146.1 & $\ldots$ & 130 & & & 101.2 & 171.2 & $\ldots$ & $\ldots$ & $\ldots$ & $\ldots$ & $\ldots$ & $\ldots$ & 94.5 & 161.5 & 90.3 & & 117.5 & $\ldots$ & $\ldots$ \\
\hline $4415.14 \ldots \ldots$. & $\mathrm{Fe}_{\mathrm{I}}$ & 1.61 & -0.621 & 104 & 126.4 & 126.5 & & 109 & 134.8 & 108 & 82.2 & 136.7 & & 102.2 & 94 & 100.3 & & 69.6 & 76.7 & 135.6 & 82.3 & 111.7 & 101.7 & & \\
\hline $4430.62 \ldots \ldots$ & $\mathrm{Fe}_{\mathrm{I}}$ & 2.22 & -1.728 & 26.7 & 55.7 & 39.1 & 86.5 & 31.3 & 54.7 & 27 & 13.8 & 61.8 & 28 & $\ldots$ & 19.2 & $\cdots$ & 39.1 & $\ldots$ & $\ldots$ & $\ldots$ & & 26.9 & 22.4 & 40.1 & \\
\hline $4442.35 \ldots \ldots$ & $\mathrm{Fe}_{\mathrm{I}}$ & 2.20 & -1.228 & 49.3 & 82.2 & 66.7 & 118.1 & 52.7 & 78.8 & 51.8 & 29.2 & 88.7 & 52.8 & 41 & 36.6 & 32.8 & 59.7 & 19 & 22.4 & $\ldots$ & 32.8 & 61.7 & 34.8 & 68.1 & 66.2 \\
\hline $4443.20 \ldots \ldots$ & $\mathrm{Fe}_{\mathrm{I}}$ & 2.86 & -1.043 & 18.9 & 41.3 & 33.7 & & 18.7 & 37.4 & 19.2 & 12.3 & 47 & 19.9 & 14.3 & 13 & 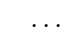 & 25.2 & & 9.1 & 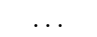 & & 15.3 & 18.2 & 29.4 & \\
\hline $4447.73 \ldots \ldots$ & $\mathrm{Fe}_{\mathrm{I}}$ & 2.22 & -1.339 & 44.7 & 74.2 & 62.4 & 104.6 & 45.6 & 72.6 & 44 & 24.5 & 80.4 & 42.1 & 35.6 & 27.6 & 23 & 53.5 & 17.1 & 16.8 & & 21.2 & 44.2 & 33.9 & 57.8 & 52.9 \\
\hline $4466.56 \ldots \ldots$ & $\mathrm{Fe}_{\mathrm{I}}$ & 2.83 & -0.600 & 49.3 & 83.6 & 71.8 & & 54.7 & 84.5 & 50.7 & 28.9 & 90.3 & 48.8 & 41 & 36.2 & 28.6 & 63.7 & 19.3 & 23.4 & & 29.9 & & & 65.1 & 62.4 \\
\hline $4489.75 \ldots \ldots$ & $\mathrm{Fe} \mathrm{I}$ & 0.12 & -3.899 & 40.3 & 82.1 & 82.8 & 136.2 & 52.8 & 84.9 & 46.1 & 11.6 & 85.9 & 44.1 & 35.1 & 32.5 & 5.6 & 64.3 & $\ldots$ & 9 & $\ldots$ & $\ldots$ & 43.6 & 26.2 & 56.4 & 55.3 \\
\hline $4494.57 \ldots \ldots$. & $\mathrm{Fe}_{\mathrm{I}}$ & 2.20 & -1.143 & 54.9 & 85.8 & 70.2 & 120.5 & 60.3 & 82.9 & 55.7 & 34.5 & 88 & 51.4 & 44.5 & 43.9 & 41.6 & 64.9 & $\ldots$ & 27.4 & 68.8 & 31.5 & 50.3 & 35.2 & 65.8 & 65.9 \\
\hline $4528.63 \ldots \ldots$. & $\mathrm{Fe}_{\mathrm{I}}$ & 2.18 & -0.887 & 71 & 112.9 & 90.6 & 153.3 & 77.4 & 103.3 & 73.4 & 51 & 114.9 & 65.9 & 63.5 & 55.1 & 57.2 & 76.4 & 32.6 & 42.8 & 90.8 & 40.7 & 79.5 & 60.7 & 89.6 & $\ldots$ \\
\hline $4531.16 \ldots \ldots$. & $\mathrm{Fe}_{\mathrm{I}}$ & 1.49 & -2.101 & 48.9 & 82.4 & 68 & 127 & 55.8 & 82.6 & 50.6 & 21.9 & 87.6 & 47.5 & 39.3 & 37.4 & 31.7 & $\ldots$ & $\ldots$ & $\ldots$ & 70.1 & 25.3 & 49.4 & 38.3 & 63.1 & 60.7 \\
\hline $4602.95 \ldots \ldots$. & $\mathrm{Fe}_{\mathrm{I}}$ & 1.49 & -2.208 & 46.3 & 81.2 & 71.6 & 121.1 & 53.1 & 80.1 & 48.4 & 20.2 & 27.2 & 44 & 39.1 & 32.8 & 30.8 & 60.7 & 22.3 & 19.8 & 65.9 & & 43.7 & 32.7 & 59.8 & 57 \\
\hline $4736.78 \ldots \ldots$ & $\mathrm{Fe}_{\mathrm{I}}$ & 3.21 & -0.752 & 23.1 & 45.6 & 32.7 & 72.6 & 23.4 & 43.1 & 22.5 & 14.2 & 53. & 24.4 & 16.8 & 16 & 12. & 24.6 & 14.4 & $\ldots$ & 38.8 & & 22.5 & $\cdots$ & 33.2 & 29 \\
\hline $4871.32 \ldots \ldots$. & $\mathrm{Fe}_{\mathrm{I}}$ & 2.87 & -0.362 & 51.2 & 82.5 & 64.5 & 107.9 & 55.7 & 79 & 52 & 36.3 & 86.5 & 54.6 & 44.4 & 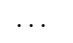 & 37.6 & 59.5 & 23.5 & 33.2 & 69.2 & 33.1 & 54.9 & 39.2 & 65.2 & 63.8 \\
\hline $4872.14 \ldots \ldots$. & $\mathrm{Fe}_{\mathrm{I}}$ & 2.88 & -0.567 & 41.5 & 71.9 & 55.6 & 99.8 & 45.6 & 67.7 & 42.8 & 27.1 & 75.8 & 44 & 32.9 & $\ldots$ & 29.7 & 46.3 & 21.7 & 27.4 & 56 & 21.3 & 41.3 & 29.1 & 53.6 & 54.3 \\
\hline $4890.76 \ldots \ldots$. & $\mathrm{Fe} \mathrm{I}_{\mathrm{I}}$ & 2.88 & -0.394 & 51 & 84.5 & 62.3 & 109.2 & 54.9 & 77.8 & 50.6 & 35.7 & 86.7 & 50.5 & 44.3 & 33.1 & 32.3 & 60.4 & 19.4 & 30 & 69.8 & & 56.9 & 37.6 & 65.2 & \\
\hline $4891.50 \ldots \ldots$ & $\mathrm{Fe}_{\mathrm{I}}$ & 2.85 & -0.111 & 64.5 & 93.2 & 79 & 121.4 & 67.8 & 91.6 & 64 & 49.5 & 97.7 & 66.6 & 58.7 & 46.5 & 48.8 & 71.4 & 30.8 & 43.7 & 80.5 & 42.3 & 61.5 & 50 & 76.8 & 74.1 \\
\hline $4903.32 \ldots \ldots$. & $\mathrm{Fe}_{\mathrm{I}}$ & 2.88 & -0.926 & 25.6 & 55 & 37.4 & 80.7 & 28.4 & 50.4 & 26.8 & 15.9 & 57.2 & 27.3 & 20 & 17.4 & 15.3 & 29.8 & $\ldots$ & 15 & 41.6 & 18 & 25.2 & 21.9 & 40.8 & 35.3 \\
\hline $4919.00 \ldots \ldots$. & $\mathrm{Fe}_{\mathrm{I}}$ & 2.87 & -0.342 & 52.7 & 83.7 & 66.9 & 110.7 & 56.4 & 78.9 & 53 & 38.2 & 86.9 & 54 & 44.9 & 39.5 & 37.1 & 62.6 & 28.7 & 33.2 & 65.1 & 30.8 & 55.8 & 42.5 & 68.3 & 64.2 \\
\hline $4924.77 \ldots \ldots .$. & $\mathrm{Fe}_{\mathrm{I}}$ & 2.28 & -2.200 & 10.1 & 29.1 & 23.6 & 60.3 & 14.5 & 29.7 & 11.8 & 4.1 & 36.2 & $\ldots$ & $\ldots$ & 7.6 & $\ldots$ & $\ldots$ & $\ldots$ & $\ldots$ & 19.8 & $\ldots$ & 10.6 & $\ldots$ & 19 & 19.3 \\
\hline $4938.82 \ldots \ldots$. & $\mathrm{Fe}_{\mathrm{I}}$ & 2.88 & -1.077 & 18.5 & 45.6 & 32.1 & 72.3 & 22.4 & 42.8 & 21.8 & 11.7 & 51.8 & 22.8 & 16 & 9.9 & 12.3 & 31.9 & 5.9 & & & & 20.1 & 16.5 & 35.2 & 26.4 \\
\hline $4939.69 \ldots \ldots$. & $\mathrm{Fe}_{\mathrm{I}}$ & 0.86 & -3.252 & 30 & 67.8 & 63.1 & 117.4 & 37.9 & 70.7 & 33.6 & 8.6 & 77.1 & 32.4 & 26.6 & 17.8 & 20.8 & 50 & & & 55.9 & & & 21.2 & 47.1 & 39.8 \\
\hline
\end{tabular}


TABLE 3-Continued

\begin{tabular}{|c|c|c|c|c|c|c|c|c|c|c|c|c|c|c|c|c|c|c|c|c|c|c|c|c|c|}
\hline \multirow{2}{*}{$\begin{array}{l}\text { WAVELENGTH } \\
(\AA)\end{array}$} & \multirow{2}{*}{\multicolumn{2}{|c|}{$\begin{array}{r}\text { L.E.P. } \\
(\mathrm{eV})\end{array}$}} & \multirow[b]{2}{*}{$\log g f$} & \multicolumn{22}{|c|}{ Equivalent Width $(\mathrm{m} \AA)^{\mathrm{a}}$} \\
\hline & & & & 1 & 2 & 3 & 4 & 5 & 6 & 7 & 8 & 9 & 10 & 11 & 12 & 13 & 14 & 15 & 16 & 17 & 18 & 19 & 20 & 21 & 22 \\
\hline $4946.39 \ldots \ldots .$. & $\mathrm{Fe}_{\mathrm{I}}$ & 3.37 & -1.010 & 7.2 & 19.6 & & 35.3 & 6.8 & 16.5 & 7.4 & 3.9 & 21.4 & & & & & & & & $\ldots$ & $\ldots$ & & & 15.9 & \\
\hline $66.09 \ldots \ldots$ & $\mathrm{Fe}_{\mathrm{I}}$ & 33 & 0.840 & 13.9 & 34.5 & 21.7 & 56.2 & 15.4 & 31.1 & 14.5 & & 39 & 14 & 10.8 & $\ldots$ & 8.7 & 20.8 & & $\ldots$ & $\ldots$ & $\ldots$ & 12 & 11.2 & 23.9 & 17 \\
\hline $73.01 \ldots \ldots$ & $\mathrm{Fe}_{\mathrm{I}}$ & 3.96 & -0.850 & 3.8 & 9.3 & 3.2 & 16.8 & 4.6 & 9.7 & 3.3 & 2.7 & 9.8 & & & & & & & & & & & & & \\
\hline $4994.14 \ldots \ldots .$. & $\mathrm{Fe}_{\mathrm{I}}$ & 0.92 & -2.969 & 39.8 & 79.4 & 70.5 & 127 & 50.1 & 81.9 & 43 & 13 & 83.3 & 42 & 31.8 & 27.8 & 31 & 59.9 & 17.4 & $\cdots$ & 60.3 & 18.8 & 41.4 & 32.3 & 50.7 & 56 \\
\hline $5001.87 \ldots \ldots .$. & $\mathrm{Fe}_{\mathrm{I}}$ & 3.88 & 0.050 & 16.6 & 35.7 & 24.2 & 51.1 & 15.4 & 30.3 & 16.3 & 13.2 & 40.3 & 17.3 & 6.1 & 10.8 & 10.6 & 23.9 & 9.1 & 14.3 & 29.3 & & 18.1 & & 28.8 & 20.2 \\
\hline $5006.12 \ldots \ldots$. & $\mathrm{Fe}_{\mathrm{I}}$ & 83 & -0.615 & 42.5 & 73 & 58.6 & 101.7 & 45.2 & 70 & 43.1 & 27.2 & 78.8 & 39.3 & 33.5 & 28.8 & 28.4 & 49.8 & 15.5 & 23.4 & $\ldots$ & 23.6 & 41.1 & 27.5 & 57.6 & 51.4 \\
\hline $5014.94 \ldots \ldots$. & $\mathrm{Fe}_{\mathrm{I}}$ & 3.94 & -0.270 & 10.2 & 23.7 & 12.2 & 37.5 & 13 & 22.5 & 10.1 & 8 & 26.7 & $\ldots$ & 11.1 & $\ldots$ & $\ldots$ & 13 & $\ldots$ & $\ldots$ & $\ldots$ & $\ldots$ & 6.4 & $\ldots$ & 16.2 & 13.3 \\
\hline $5022.24 \ldots \ldots$. & $\mathrm{Fe}_{\mathrm{I}}$ & 98 & -0.490 & 6.4 & 16.3 & 9.8 & 26.5 & 8.5 & 14.4 & 7.4 & 5.6 & 18.9 & $\ldots$ & & $\ldots$ & $\ldots$ & $\ldots$ & & $\ldots$ & $\ldots$ & $\ldots$ & $\ldots$ & $\ldots$ & 14.5 & 6.2 \\
\hline $5041.76 \ldots \ldots$ & $\mathrm{Fe}_{\mathrm{I}}$ & 9 & -2.2 & 52.1 & 95.3 & 82.4 & 141.9 & 56.1 & 91.7 & 53.8 & 22.1 & 101.4 & 53.5 & 47.1 & 36.4 & 33.2 & 61.7 & 19.7 & 22.8 & $\ldots$ & 25.7 & 49.8 & 30.6 & 67.6 & $\ldots$ \\
\hline $5044.21 \ldots \ldots$. & $\mathrm{Fe}_{\mathrm{I}}$ & & -2.040 & 3.8 & 11.4 & 6.7 & 23. & 5.1 & 9. & 3. & 1.7 & & & & & $\ldots$ & & & $\ldots$ & $\ldots$ & $\ldots$ & $\ldots$ & $\ldots$ & $\ldots$ & \\
\hline $5049.83 \ldots \ldots$ & $\mathrm{Fe}_{\mathrm{I}}$ & 2 & 1.355 & 39.5 & 73.2 & 62 & 109 & 43 & 72.3 & 42.2 & 21.5 & 79.4 & 40.4 & 32.5 & 25.3 & 27 & 49.8 & 13.3 & 20.4 & 54.9 & 21.9 & 38.3 & 24 & 56.4 & 51.8 \\
\hline $5051.64 \ldots \ldots$. & $\mathrm{Fe}_{\mathrm{I}}$ & 0 & -2.764 & 56.9 & 96.7 & 90.5 & 145.8 & 67 & 97.6 & 61. & 23.9 & 101.8 & 57 & 51 & 41. & 44 & 76.7 & 21.3 & 21.7 & 83 & 32.2 & 56.6 & 41.6 & 71.4 & 71.6 \\
\hline $5068.77 \ldots \ldots$. & $\mathrm{Fe}_{\mathrm{I}}$ & 2.94 & -1.041 & 17.1 & 42.7 & 26.2 & 68.1 & 21.7 & 39.3 & 19.7 & 11.7 & 48.3 & & 15.7 & 17.5 & 14.2 & 25.6 & & & 39.1 & & 19.2 & & 29.1 & 27.9 \\
\hline $5074.75 \ldots \ldots .$. & $\mathrm{Fe}_{\mathrm{I}}$ & 4.22 & -0.160 & 8 & 21.7 & 12 & 31.7 & 9.5 & 18 & 8. & 7.9 & 23.7 & $\ldots$ & & $\ldots$ & $\ldots$ & $\ldots$ & & $\ldots$ & $\ldots$ & $\ldots$ & 7.5 & $\ldots$ & 17 & $\ldots$ \\
\hline $5083.35 \ldots \ldots$ & $\mathrm{Fe}_{\mathrm{I}}$ & 0.96 & -2.842 & 44.1 & 83.5 & 76.6 & 129.7 & 53.7 & 84.7 & 48.3 & 15.5 & 89.8 & 43.3 & 39.6 & 29.8 & 31.3 & 63.3 & 14.4 & 13.2 & 67.6 & & 41.9 & 28.2 & 58.5 & 59.1 \\
\hline $5090.77 \ldots \ldots$. & $\mathrm{Fe}_{\mathrm{I}}$ & 4.26 & -0.360 & 4.5 & 8.5 & $\ldots$ & 15 & 4 & 7.2 & 3.8 & 2.4 & 9.7 & $\ldots$ & & $\ldots$ & $\ldots$ & $\ldots$ & $\ldots$ & $\ldots$ & $\ldots$ & $\ldots$ & $\ldots$ & $\ldots$ & 6.6 & $\ldots$ \\
\hline $5123.73 \ldots \ldots$. & $\mathrm{Fe}_{\mathrm{I}}$ & 1.01 & -3.057 & $\ldots$ & $\ldots$ & $\ldots$ & 126.7 & $\ldots$ & 77.5 & 38.6 & $\ldots$ & $\ldots$ & 35.7 & 30.8 & 22.6 & 26.6 & 54.3 & $\ldots$ & $\ldots$ & $\ldots$ & $\ldots$ & 47.6 & $\ldots$ & 53.6 & $\ldots$ \\
\hline $5125.12 \ldots \ldots$. & $\mathrm{Fe} \mathrm{I}$ & 4. & -0.080 & $\ldots$ & $\ldots$ & $\ldots$ & 35.4 & $\ldots$ & 19.5 & 8.3 & $\ldots$ & $\ldots$ & $\ldots$ & $\ldots$ & $\ldots$ & $\ldots$ & $\ldots$ & $\ldots$ & $\ldots$ & $\ldots$ & $\ldots$ & $\ldots$ & $\ldots$ & 16.2 & $\ldots$ \\
\hline $5127.37 \ldots \ldots$. & $\mathrm{Fe}_{\mathrm{I}}$ & 0.92 & -3.24 & $\ldots$ & $\ldots$ & $\ldots$ & 117.4 & $\ldots$ & 70.5 & 32.8 & $\ldots$ & $\ldots$ & $\ldots$ & 26.5 & 19.7 & 17.6 & 47.7 & $\ldots$ & $\ldots$ & $\ldots$ & $\ldots$ & 30.3 & $\ldots$ & 40.4 & $\cdots$ \\
\hline $5133.69 \ldots \ldots$ & $\mathrm{Fe}_{\mathrm{I}}$ & 4 & 0.20 & $\ldots$ & $\ldots$ & $\ldots$ & 51 & $\ldots$ & 31. & & $\ldots$ & $\ldots$ & $\ldots$ & 12.8 & & & 20.2 & & $\ldots$ & $\ldots$ & $\ldots$ & 18 & $\ldots$ & 27.2 & 19.9 \\
\hline $5150.85 \ldots \ldots$ & $\mathrm{Fe}_{\mathrm{I}}$ & 0.99 & -3.037 & $\ldots$ & $\ldots$ & $\ldots$ & 118 & $\ldots$ & 73. & 34 & $\ldots$ & $\ldots$ & 30.7 & 25. & 22 & 20.2 & 50.1 & 9.7 & $\ldots$ & $\ldots$ & $\ldots$ & 33.3 & $\ldots$ & 47.3 & 45.6 \\
\hline $5151.92 \ldots \ldots$ & $\mathrm{Fe}_{\mathrm{I}}$ & 1.01 & -3.321 & $\ldots$ & $\ldots$ & $\ldots$ & 110.5 & $\ldots$ & 61.5 & 25. & $\ldots$ & $\ldots$ & 23.3 & 20. & 15.1 & 16.8 & 40.3 & & $\ldots$ & $\ldots$ & $\ldots$ & 27.4 & $\ldots$ & 35.4 & 30.8 \\
\hline $5162.28 \ldots \ldots$. & $\mathrm{Fe}_{\mathrm{I}}$ & & & $\ldots$ & $\ldots$ & $\ldots$ & & $\ldots$ & 27 & & $\ldots$ & $\ldots$ & $\ldots$ & 14 & 11 & 10.1 & 22 & & $\ldots$ & $\ldots$ & $\ldots$ & $\ldots$ & $\ldots$ & 26.1 & 19.7 \\
\hline $5171.61 \ldots \ldots$. & $\mathrm{Fe}_{\mathrm{I}}$ & & -1.72 & $\ldots$ & $\ldots$ & $\ldots$ & 147.3 & $\ldots$ & 107.4 & 75.4 & $\ldots$ & $\ldots$ & 69.8 & 68 & 57 & 58.3 & 86.3 & & $\ldots$ & $\ldots$ & $\ldots$ & 69.9 & $\ldots$ & 80.1 & 87 \\
\hline $5191.47 \ldots \ldots$. & $\mathrm{Fe}_{\mathrm{I}}$ & 3.04 & -0.551 & $\ldots$ & $\ldots$ & $\ldots$ & 100.5 & $\ldots$ & 60.2 & 34 & $\ldots$ & $\ldots$ & 31.2 & 24.7 & 21.6 & 19.7 & 37.8 & 10.4 & $\ldots$ & $\ldots$ & & $\ldots$ & $\ldots$ & 48.9 & $\ldots$ \\
\hline $5192.35 \ldots \ldots$ & $\mathrm{Fe}_{\mathrm{I}}$ & 3.00 & -0.421 & $\ldots$ & $\ldots$ & $\ldots$ & 99.7 & $\ldots$ & 70.3 & 41.7 & $\ldots$ & $\ldots$ & 41.1 & 34.1 & 26.2 & 26 & 48.1 & 17 & $\ldots$ & $\ldots$ & $\ldots$ & $\ldots$ & $\ldots$ & 53.9 & 51.7 \\
\hline $5194.95 \ldots \ldots .$. & $\mathrm{Fe}_{\mathrm{I}}$ & 1. & -2.021 & $\ldots$ & $\ldots$ & $\ldots$ & 13.1 & $\ldots$ & 89.6 & 54.5 & $\ldots$ & $\ldots$ & 51.6 & 44.6 & 39.7 & 37.8 & 67.7 & 17.1 & $\ldots$ & $\ldots$ & $\ldots$ & 53.3 & $\ldots$ & 65.2 & 67.7 \\
\hline $.71 \ldots \ldots$. & $\mathrm{Fe}_{\mathrm{I}}$ & & -2.1 & $\ldots$ & $\ldots$ & $\ldots$ & 130.1 & $\ldots$ & 38 & 14.4 & $\ldots$ & $\ldots$ & 16 & 11 & 11.7 & $\ldots$ & $\ldots$ & $\ldots$ & $\ldots$ & $\ldots$ & $\ldots$ & 14 & $\ldots$ & 23.7 & $\ldots$ \\
\hline $4178.86 \ldots \ldots$. & $\mathrm{Fe}$ II & 2.58 & -2.489 & 41.2 & 63.9 & 47.7 & $\ldots$ & 35.2 & 56.1 & 3 & 18 & 56.5 & 34.3 & 32. & 20.7 & & 43 & & & $\ldots$ & $\ldots$ & 30.2 & $\ldots$ & 43.5 & 39.7 \\
\hline $17 \ldots \ldots$ & $\mathrm{Fe}$ II & & -1.90 & $\ldots$ & $\ldots$ & $\ldots$ & $\ldots$ & $\ldots$ & 88. & 62. & 43.2 & $\ldots$ & $\ldots$ & 60 & 50.7 & 70.4 & 76.2 & . & & $\ldots$ & $\ldots$ & 66.1 & $\ldots$ & 73.4 & 75.4 \\
\hline $4416.83 \ldots \ldots$ & $\mathrm{Fe}$ II & 2 & -2.580 & 21.2 & 53.9 & 30.3 & 64.8 & 26.6 & 41.4 & 20. & 12 & 51.6 & & 15. & 16.4 & & & & 13.6 & $\ldots$ & $\ldots$ & 26.8 & 16.4 & 35.4 & \\
\hline $4491.41 \ldots \ldots .$. & $\mathrm{Fe}$ II & & -2.5 & 15.8 & 35.5 & 24. & 46 & & 30 & 13 & 7.6 & 36.1 & 17.2 & 11 & & 8.6 & 26.9 & & 4.3 & $\ldots$ & $\ldots$ & 14 & 14.4 & 24.2 & 16.3 \\
\hline $4508.29 \ldots \ldots$. & $\mathrm{Fe}$ II & & -2.31 & 26.6 & 62.9 & 40.7 & 71.9 & 32.1 & 51.2 & 2 & 16.1 & 57. & 29.9 & & 20.4 & 18.4 & 42.2 & 12 & 14.2 & 50.5 & 13.2 & 28.4 & 22 & 42.7 & 37.7 \\
\hline $4515.34 \ldots \ldots .$. & $\mathrm{Fe}_{\mathrm{II}}$ & 2.84 & -2.422 & 22.3 & 53.5 & 34.8 & $\ldots$ & 25.7 & 43.9 & 23 & 12.6 & 51.3 & 25.1 & 22.1 & 13.6 & 16 & 32.5 & & 9.7 & 37.9 & 9.5 & 22.4 & 15.8 & 31.7 & 28.3 \\
\hline $4520.23 \ldots \ldots .$. & $\mathrm{Fe}$ II & 2.81 & -2.590 & 21.3 & 51 & 28.8 & $\ldots$ & 25.3 & 42.8 & 22.1 & 13.2 & 48.5 & 24.3 & 19.6 & 15.9 & 12.3 & 27.7 & & 9.3 & 39.7 & $\ldots$ & 22.2 & 14.8 & 27.8 & $\ldots$ \\
\hline $4522.64 \ldots \ldots$ & $\mathrm{Fe}$ II & 2.84 & -2.050 & & & $\ldots$ & $\ldots$ & 51.4 & 65.6 & 42.7 & 22.8 & $\ldots$ & 42.3 & 38.9 & $\ldots$ & $\ldots$ & 54.8 & 18.7 & 17.9 & $\ldots$ & 22.3 & 57.4 & 34.1 & 56.3 & $\ldots$ \\
\hline $4541.52 \ldots \ldots .$. & $\mathrm{Fe}$ II & 2.86 & -3.030 & 7.7 & 27.3 & 18.8 & $\ldots$ & 11.8 & 21.9 & 10.7 & $\ldots$ & 28.8 & $\ldots$ & $\ldots$ & $\ldots$ & $\ldots$ & $\ldots$ & $\ldots$ & $\ldots$ & $\ldots$ & $\ldots$ & 17.8 & $\ldots$ & 14.3 & \\
\hline $4555.89 \ldots \ldots .$. & $\mathrm{Fe}_{\text {II }}$ & & -2.30 & 26.7 & $\ldots$ & 39.7 & $\ldots$ & 31.9 & 53.1 & 30. & $\ldots$ & 59.7 & & & & & & & 15.1 & $\ldots$ & & 25.6 & $\ldots$ & 37.8 & 35.8 \\
\hline $4576.34 \ldots \ldots .$. & $\mathrm{Fe}$ II & & -2.920 & 9 & 28.6 & 17.4 & 39.9 & 12.6 & 23.3 & 11 & $\ldots$ & 29.4 & & 10.9 & & $\ldots$ & & & $\cdots$ & $\ldots$ & $\ldots$ & $\ldots$ & $\ldots$ & 18.9 & 16.2 \\
\hline $583.84 \ldots \ldots$. & e II & & -1.89 & 53.2 & 92.4 & 73.8 & 104.8 & & 16.9 & 56 & $\ldots$ & 86.2 & 52.8 & 55 & 40.5 & 41.9 & 67.3 & 22.5 & 32.6 & 76.6 & 29.5 & 53.9 & 50.8 & 66.2 & 64.6 \\
\hline $4923.93 \ldots \ldots$ & $\mathrm{Fe}$ II & & -1.30 & 73.7 & 114.4 & 93.7 & 126.4 & 82.3 & 104.2 & 76. & 56.9 & 106.7 & 73.3 & 79.5 & 63.2 & 70 & 84.8 & 39.1 & 49.1 & 92.2 & 46.2 & 76.8 & 67.9 & 86 & 86.9 \\
\hline $4993.35 \ldots \ldots .$. & e II & & -3.6 & 1.8 & 8.4 & 4.7 & & 4.2 & 8.8 & 3. & 1 & 9.4 & & & & . & & & 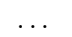 & $\ldots$ & & $\ldots$ & $\ldots$ & 3.1 & \\
\hline $5018.45 \ldots \ldots$. & $\mathrm{Fe}$ II & 2.89 & -1.292 & 84.8 & 128.1 & 106.3 & 143.6 & 88.4 & 115.1 & 86.1 & 67.5 & 117.5 & 80.2 & 89.2 & 71.5 & 77.3 & 92.8 & 47 & 57.5 & 106.4 & 54.5 & 83.2 & 79.5 & 93.9 & 95.9 \\
\hline $5197.58 \ldots \ldots$ & $\mathrm{Fe}$ II & 3.23 & -2.167 & $\ldots$ & $\ldots$ & $\ldots$ & 54.8 & $\ldots$ & 39.4 & 19.3 & $\ldots$ & $\ldots$ & $\ldots$ & 17.2 & 12.2 & & 28.3 & & $\ldots$ & $\ldots$ & $\ldots$ & $\ldots$ & $\ldots$ & 29.3 & \\
\hline $3845.47 \ldots \ldots .$. & Co I & 0.92 & 0.010 & 64.8 & 91.7 & 58.7 & 108.1 & 69.3 & 92 & 67.6 & 39.6 & 87.5 & 66.1 & 64.4 & 59.3 & 48.2 & 68.3 & 36.4 & 37.1 & 73 & 39.3 & 63.6 & 54.5 & 70.9 & 73.4 \\
\hline $3995.32 \ldots \ldots .$. & Co I & 0.92 & -0.220 & $\ldots$ & $\ldots$ & $\ldots$ & $\ldots$ & 61.7 & 80.4 & 59.6 & 32.8 & $\cdots$ & 54.2 & 52.2 & 48.6 & 49.3 & 58 & 32.7 & 29.1 & 51.3 & 37.2 & 57 & $\cdots$ & 66.6 & 68 \\
\hline
\end{tabular}


TABLE 3-Continued

\begin{tabular}{|c|c|c|c|c|c|c|c|c|c|c|c|c|c|c|c|c|c|c|c|c|c|c|c|c|c|}
\hline \multirow{2}{*}{$\begin{array}{c}\text { WAVELENGTH } \\
(\AA)\end{array}$} & \multirow[b]{2}{*}{ SPECIES } & \multirow{2}{*}{$\begin{array}{r}\text { L.E.P. } \\
(\mathrm{eV})\end{array}$} & \multirow[b]{2}{*}{$\log g f$} & \multicolumn{22}{|c|}{ EQUivalent WidTh $(\mathrm{m} \AA)^{\mathrm{a}}$} \\
\hline & & & & 1 & 2 & 3 & 4 & 5 & 6 & 7 & 8 & 9 & 10 & 11 & 12 & 13 & 14 & 15 & 16 & 17 & 18 & 19 & 20 & 21 & 22 \\
\hline $4118.78 \ldots \ldots$. & Co I & 1.05 & -0.490 & & $\ldots$ & $\ldots$ & $\ldots$ & $\ldots$ & $\ldots$ & $\ldots$ & 18 & & & $\ldots$ & $\ldots$ & $\ldots$ & $\ldots$ & $\ldots$ & $\ldots$ & $\ldots$ & 20.5 & $\ldots$ & $\ldots$ & $\ldots$ & \\
\hline $4121.32 \ldots \ldots$ & Co I & 0.92 & -0.320 & 62.9 & 88.3 & 72.6 & 121 & 65.9 & 90 & 64.1 & 30.4 & 91.5 & 61.3 & 56.6 & 50.7 & 54.9 & 63.4 & 26.5 & 27.8 & 56.8 & 38.3 & Syn & 41.6 & 71.4 & 73.3 \\
\hline $3807.15 \ldots \ldots$ & $\mathrm{Ni}$ I & 0.42 & -1.180 & 85.3 & 111.9 & 111 & 145 & 91.3 & 116.3 & 86.3 & 64.3 & 113.4 & 83.3 & 93.1 & 71.7 & 83.1 & 85.6 & 52.3 & 59.1 & 114.9 & 70.3 & 81.5 & 72.9 & 90 & \\
\hline $3858.30 \ldots \ldots$ & $\mathrm{Ni}$ I & 0.42 & -0.970 & 93 & 123.3 & 91.4 & 178.7 & 97.6 & 125.6 & 95.5 & 72.1 & 121.4 & 78.7 & 97.9 & 84.3 & 89.5 & 87 & 61.9 & 63.8 & 78.5 & 61.7 & 90.7 & 85.3 & 93.9 & 106.9 \\
\hline $5105.55 \ldots \ldots$. & $\mathrm{Cu}$ I & 1.39 & -1.520 & & & 1.8 & 19.5 & $\ldots$ & 3.3 & & & 5.2 & & & & & & $\ldots$ & $\ldots$ & $\ldots$ & $\ldots$ & & & & \\
\hline $4722.16 \ldots \ldots$ & $\mathrm{Zn}$ I & 4.03 & -0.390 & 7.8 & 21.3 & 10.5 & 29.8 & 8.8 & 15.1 & 7.3 & 3.1 & 20.6 & 11.6 & 7.6 & 7.3 & 20.5 & 10.1 & $\ldots$ & $\ldots$ & $\ldots$ & $\ldots$ & 14.1 & 11.2 & 13.6 & 8.3 \\
\hline $4810.54 \ldots \ldots$. & $\mathrm{Zn}$ I & 4.08 & -0.170 & 11.6 & 23.4 & 14.2 & 35.6 & 11.7 & 20.5 & 11 & 5 & 25.6 & 8.9 & 8 & 11.9 & $\ldots$ & 14.5 & $\ldots$ & $\ldots$ & $\ldots$ & $\ldots$ & 9.2 & 8.5 & & 12.2 \\
\hline $4077.72 \ldots \ldots$ & $\mathrm{Sr}$ II & 0.00 & 0.150 & 121 & Syn & 158.2 & Syn & $\ldots$ & $\ldots$ & $\ldots$ & $\ldots$ & Syn & $\ldots$ & $\ldots$ & $\ldots$ & $\ldots$ & $\ldots$ & 94.4 & 89.9 & $\ldots$ & $\ldots$ & Syn & 100 & Syn & Syn \\
\hline $4161.82 \ldots \ldots \ldots$ & $\mathrm{Sr}$ II & 2.94 & -0.600 & & 7.4 & $\ldots$ & $\ldots$ & 1.6 & $\ldots$ & $\ldots$ & $\ldots$ & & & $\ldots$ & & $\ldots$ & $\ldots$ & $\ldots$ & $\ldots$ & 134.8 & $\ldots$ & $\ldots$ & $\ldots$ & & \\
\hline $4215.54 \ldots \ldots$. & $\mathrm{Sr}$ II & 0.00 & -0.170 & & 172 & 147 & 240.1 & 129.6 & 159.6 & 124.7 & 66.1 & 172.6 & 84.1 & 46.1 & 105.1 & 86 & 118 & 81.5 & $\ldots$ & 93.7 & 78.8 & 146.4 & 73.9 & 141.3 & 163.1 \\
\hline $3774.33 \ldots \ldots$ & Y II & 0.13 & 0.210 & 47.7 & 88.6 & $\ldots$ & 120.3 & 64.1 & $\ldots$ & $\ldots$ & $\ldots$ & 89 & & $\ldots$ & & $\ldots$ & $\ldots$ & $\ldots$ & 20.6 & $\ldots$ & $\ldots$ & 82.7 & $\ldots$ & 68.8 & 89.5 \\
\hline $3788.70 \ldots \ldots$ & Y II & 0.10 & -0.070 & 40.2 & 82.7 & 85.8 & 111.5 & 58.3 & 61.7 & 47 & 6.3 & 79.6 & 17.7 & $\ldots$ & 37.4 & $\ldots$ & 50.8 & 31.8 & 19.8 & $\ldots$ & 35.1 & 72.3 & $\ldots$ & 59.4 & 88.6 \\
\hline $3818.34 \ldots \ldots$. & Y II & 0.13 & -0.980 & & Syn & $\ldots$ & $\ldots$ & 20.5 & 29.4 & 14.6 & $\ldots$ & $\ldots$ & 8 & $\ldots$ & 8.2 & $\ldots$ & 18.5 & $\ldots$ & $\ldots$ & $\ldots$ & $\ldots$ & 32.4 & $\ldots$ & 30 & 54.2 \\
\hline $3950.36 \ldots \ldots$. & Y II & 0.10 & -0.490 & 22.1 & 67.1 & 41.3 & 88.7 & 39.3 & 47.4 & 30.2 & 4.8 & 65.1 & 10.9 & & 22.3 & $\ldots$ & 25.3 & 8.2 & 9.4 & $\ldots$ & 12.8 & 57.3 & $\ldots$ & 44.7 & 71.8 \\
\hline $4177.54 \ldots \ldots$. & Y II & 0.41 & -0.160 & $\ldots$ & Syn & $\ldots$ & $\ldots$ & $\ldots$ & $\ldots$ & $\ldots$ & $\ldots$ & $\ldots$ & $\ldots$ & $\ldots$ & $\ldots$ & $\ldots$ & $\ldots$ & $\ldots$ & $\ldots$ & $\ldots$ & $\ldots$ & 81.1 & $\ldots$ & $\ldots$ & $\cdots$ \\
\hline $4398.01 \ldots \ldots$. & Y II & 0.13 & -1.000 & 10.8 & 40.9 & 26.2 & $\ldots$ & 19.7 & 30.3 & $\ldots$ & $\ldots$ & $\ldots$ & & $\cdots$ & & $\ldots$ & & $\ldots$ & $\ldots$ & $\ldots$ & $\ldots$ & $\ldots$ & $\ldots$ & & \\
\hline $4883.69 \ldots \ldots$ & Y II & 1.08 & 0.070 & 7 & 38.6 & 22.3 & 59.4 & 16.2 & 25 & 11.3 & 1 & 41.4 & 7.3 & $\ldots$ & 9 & $\ldots$ & 14.4 & $\ldots$ & $\ldots$ & $\ldots$ & $\ldots$ & 34.6 & $\ldots$ & 24.6 & 41.3 \\
\hline $5087.43 \ldots \ldots$ & Y II & 1.08 & -0.170 & 4.2 & 24.4 & 13.7 & 44.2 & 9 & 13.8 & 6 & $\ldots$ & 26.7 & & $\ldots$ & & $\ldots$ & 10.5 & $\ldots$ & $\ldots$ & $\ldots$ & $\ldots$ & 22.6 & $\ldots$ & 13.8 & 32 \\
\hline $3836.77 \ldots \ldots .$. & $\mathrm{Zr}$ II & 0.56 & -0.060 & 25.4 & 52.6 & 50.6 & $\ldots$ & 34.7 & 47.3 & $\ldots$ & 3.9 & 57.7 & 13.2 & $\ldots$ & 26.2 & $\ldots$ & 25.6 & 8.5 & $\ldots$ & $\ldots$ & $\ldots$ & 50.5 & $\ldots$ & 45.3 & 47.1 \\
\hline $4161.21 \ldots \ldots$. & $\mathrm{Zr}$ II & 0.71 & -0.720 & $\ldots$ & Syn & $\ldots$ & Syn & 15.9 & $\ldots$ & 12.2 & $\ldots$ & $\ldots$ & 5.9 & $\ldots$ & $\ldots$ & $\ldots$ & 14.5 & $\ldots$ & $\ldots$ & $\ldots$ & $\ldots$ & 30.3 & $\ldots$ & 24 & 43.2 \\
\hline $4208.99 \ldots \ldots$. & $\mathrm{Zr}$ II & 0.71 & -0.460 & 12.6 & 41.2 & 27.8 & 60.7 & 19.5 & 27 & $\ldots$ & 1.4 & 45.4 & 7.3 & $\ldots$ & 12.5 & $\ldots$ & 12 & $\ldots$ & $\ldots$ & $\ldots$ & $\ldots$ & 31.3 & $\ldots$ & 28.3 & 42.9 \\
\hline $4317.32 \ldots \ldots$. & $\mathrm{Zr}$ II & 0.71 & -1.380 & $\ldots$ & 10.5 & 7.1 & 25.6 & 5.1 & 5.3 & 2.1 & $\ldots$ & 12.7 & 3.8 & $\ldots$ & $\ldots$ & $\ldots$ & $\ldots$ & $\ldots$ & $\ldots$ & $\ldots$ & $\ldots$ & $\ldots$ & $\ldots$ & 3.7 & 14.7 \\
\hline $3799.35 \ldots \ldots$. & $\mathrm{Ru}$ I & 0.00 & -0.070 & $\ldots$ & $\ldots$ & $\ldots$ & $\ldots$ & $\ldots$ & $\ldots$ & $\ldots$ & $\ldots$ & $\ldots$ & $\ldots$ & $\ldots$ & $\ldots$ & $\ldots$ & $\ldots$ & $\ldots$ & $\ldots$ & $\ldots$ & $\ldots$ & 8.5 & $\ldots$ & $\ldots$ & $\ldots$ \\
\hline $3404.58 \ldots \ldots$. & Pd I & 0.81 & 0.320 & $\ldots$ & Syn & $\ldots$ & $\ldots$ & Syn & $\ldots$ & $\ldots$ & $\ldots$ & Syn & $\cdots$ & $\ldots$ & $\ldots$ & $\ldots$ & $\ldots$ & $\ldots$ & $\ldots$ & $\ldots$ & $\ldots$ & $\ldots$ & $\ldots$ & $\ldots$ & \\
\hline $4554.04 \ldots \ldots$. & Ba II & 0.00 & 0.170 & Syn & Syn & Syn & Syn & Syn & Syn & Syn & Syn & Syn & Syn & Syn & Syn & Syn & Syn & Syn & Syn & Syn & Syn & Syn & Syn & Syn & Syn \\
\hline $4934.10 \ldots \ldots$ & $\mathrm{Ba}$ II & 0.00 & -0.150 & Syn & Syn & Syn & Syn & Syn & Syn & Syn & Syn & Syn & Syn & Syn & Syn & Syn & Syn & Syn & Syn & Syn & Syn & Syn & Syn & Syn & Syn \\
\hline $3988.52 \ldots \ldots$. & La II & 0.40 & 0.210 & $\ldots$ & Syn & $\ldots$ & Syn & Syn & Syn & Syn & $\ldots$ & Syn & $\ldots$ & $\ldots$ & $\ldots$ & $\ldots$ & $\ldots$ & $\ldots$ & $\ldots$ & $\ldots$ & $\ldots$ & Syn & $\ldots$ & Syn & Syn \\
\hline $3995.75 \ldots \ldots$. & La II & 0.17 & -0.060 & $\ldots$ & Syn & $\ldots$ & Syn & Syn & Syn & Syn & $\ldots$ & Syn & $\ldots$ & $\ldots$ & $\ldots$ & $\ldots$ & $\ldots$ & $\ldots$ & $\ldots$ & $\ldots$ & $\ldots$ & Syn & $\ldots$ & Syn & Syn \\
\hline $4086.71 \ldots \ldots$. & La II & 0.00 & -0.070 & Syn & Syn & $\ldots$ & Syn & Syn & Syn & Syn & $\ldots$ & Syn & $\ldots$ & $\ldots$ & $\ldots$ & $\ldots$ & $\ldots$ & $\ldots$ & $\ldots$ & $\ldots$ & $\ldots$ & Syn & $\ldots$ & Syn & Syn \\
\hline $4123.23 \ldots \ldots$ & La II & 0.32 & 0.130 & $\ldots$ & Syn & $\ldots$ & Syn & Syn & Syn & Syn & $\ldots$ & Syn & $\ldots$ & $\ldots$ & $\ldots$ & $\ldots$ & $\ldots$ & $\ldots$ & $\ldots$ & $\ldots$ & $\ldots$ & Syn & $\ldots$ & Syn & Syn \\
\hline $4333.76 \ldots \ldots$. & La II & 0.17 & -0.060 & $\ldots$ & Syn & $\ldots$ & $\ldots$ & Syn & Syn & $\ldots$ & $\ldots$ & Syn & $\ldots$ & $\ldots$ & $\ldots$ & $\ldots$ & $\ldots$ & $\ldots$ & $\ldots$ & $\ldots$ & $\ldots$ & $\ldots$ & $\ldots$ & $\ldots$ & Syn \\
\hline $5123.01 \ldots \ldots$. & La II & 0.32 & -0.850 & $\ldots$ & & $\ldots$ & Syn & $\ldots$ & $\ldots$ & $\ldots$ & $\ldots$ & $\ldots$ & $\ldots$ & $\ldots$ & $\ldots$ & $\ldots$ & $\ldots$ & $\ldots$ & $\ldots$ & $\ldots$ & $\ldots$ & Syn & $\ldots$ & $\ldots$ & Syn \\
\hline $4073.47 \ldots \ldots$. & $\mathrm{Ce}$ II & 0.48 & 0.320 & $\ldots$ & 15.7 & $\ldots$ & 12.4 & 6.2 & $\ldots$ & 2.7 & $\ldots$ & 10 & $\ldots$ & $\ldots$ & $\ldots$ & $\ldots$ & $\ldots$ & $\ldots$ & $\ldots$ & $\ldots$ & $\ldots$ & $\ldots$ & $\ldots$ & 6.4 & 26.5 \\
\hline $4083.23 \ldots \ldots$ & $\mathrm{Ce}$ II & 0.70 & 0.240 & $\ldots$ & 13.6 & $\ldots$ & 8.1 & 4 & $\ldots$ & 1.7 & $\ldots$ & 7.7 & $\ldots$ & $\ldots$ & $\ldots$ & $\ldots$ & $\ldots$ & $\ldots$ & $\ldots$ & $\ldots$ & $\ldots$ & 13.8 & $\ldots$ & $\ldots$ & 20.5 \\
\hline $4127.38 \ldots \ldots .$. & $\mathrm{Ce}$ II & 0.68 & 0.240 & $\ldots$ & Syn & $\ldots$ & $\ldots$ & 3.6 & $\ldots$ & $\ldots$ & $\ldots$ & 9.8 & $\ldots$ & $\ldots$ & $\ldots$ & $\ldots$ & $\ldots$ & $\ldots$ & $\ldots$ & $\ldots$ & $\ldots$ & 11.5 & $\ldots$ & $\ldots$ & 20.4 \\
\hline $4222.60 \ldots \ldots$ & $\mathrm{Ce}$ II & 0.12 & -0.180 & $\ldots$ & 17.7 & $\ldots$ & 18.6 & 6.1 & $\ldots$ & 3 & $\ldots$ & 15.5 & $\ldots$ & $\ldots$ & $\ldots$ & $\ldots$ & $\ldots$ & $\ldots$ & $\ldots$ & $\ldots$ & $\ldots$ & 22.5 & $\ldots$ & 11.6 & 33.4 \\
\hline $4418.79 \ldots \ldots$ & $\mathrm{Ce}$ II & 0.86 & 0.310 & & 6.4 & $\ldots$ & 4.5 & 3.4 & $\ldots$ & $\ldots$ & $\ldots$ & 6.1 & $\ldots$ & & $\ldots$ & $\ldots$ & $\ldots$ & $\ldots$ & $\ldots$ & $\ldots$ & $\ldots$ & 12.9 & $\ldots$ & 10 & \\
\hline $4486.91 \ldots \ldots$. & $\mathrm{Ce}$ II & 0.30 & -0.360 & $\ldots$ & 10.7 & $\ldots$ & 12.6 & 4.5 & $\ldots$ & 2.1 & $\ldots$ & 7.9 & $\ldots$ & $\ldots$ & $\ldots$ & $\ldots$ & $\ldots$ & $\ldots$ & $\ldots$ & $\ldots$ & $\ldots$ & 15.3 & $\ldots$ & 6 & 21.5 \\
\hline $4562.37 \ldots \ldots$. & $\mathrm{Ce}$ II & 0.48 & 0.330 & $\ldots$ & 19.3 & $\ldots$ & 17.8 & 8.1 & $\ldots$ & 3.1 & $\ldots$ & 12.5 & $\ldots$ & $\ldots$ & $\ldots$ & $\ldots$ & $\ldots$ & $\ldots$ & $\ldots$ & $\ldots$ & $\ldots$ & 26.3 & $\ldots$ & 10.5 & 35.8 \\
\hline $4628.16 \ldots \ldots$ & $\mathrm{Ce}$ II & 0.52 & 0.260 & $\ldots$ & 14.5 & $\ldots$ & 13.6 & 4.7 & $\ldots$ & $\ldots$ & $\ldots$ & 9.9 & $\ldots$ & $\ldots$ & $\ldots$ & $\ldots$ & $\ldots$ & $\ldots$ & $\ldots$ & $\ldots$ & $\ldots$ & 21.7 & $\ldots$ & 5.2 & 30.6 \\
\hline $3964.81 \ldots \ldots$. & Pr II & 0.05 & 0.090 & $\ldots$ & $\ldots$ & $\ldots$ & $\ldots$ & $\ldots$ & $\ldots$ & $\ldots$ & $\ldots$ & $\ldots$ & $\ldots$ & $\ldots$ & $\ldots$ & $\ldots$ & $\ldots$ & $\ldots$ & $\ldots$ & $\ldots$ & $\ldots$ & 20.1 & $\ldots$ & $\ldots$ & $\ldots$ \\
\hline $4062.80 \ldots \ldots$ & Pr II & 0.42 & 0.660 & $\ldots$ & $\ldots$ & $\ldots$ & 12.7 & $\ldots$ & $\ldots$ & $\ldots$ & $\ldots$ & $\ldots$ & $\ldots$ & $\ldots$ & $\ldots$ & $\ldots$ & $\ldots$ & $\ldots$ & $\ldots$ & $\ldots$ & $\ldots$ & $\ldots$ & $\ldots$ & & \\
\hline $4143.14 \ldots \ldots$ & Pr II & 0.37 & 0.370 & $\ldots$ & Syn & $\ldots$ & $\ldots$ & 12 & $\ldots$ & 6.6 & $\ldots$ & $\ldots$ & $\ldots$ & $\ldots$ & $\ldots$ & $\ldots$ & $\ldots$ & $\ldots$ & $\ldots$ & $\ldots$ & $\ldots$ & $\ldots$ & $\ldots$ & 14.7 & Syn \\
\hline $4408.84 \ldots \ldots$. & $\operatorname{Pr}$ II & 0.00 & -0.010 & & 18.3 & $\ldots$ & $\ldots$ & 9.3 & $\ldots$ & $\ldots$ & $\ldots$ & 11.9 & $\ldots$ & $\ldots$ & $\ldots$ & $\ldots$ & $\ldots$ & $\ldots$ & $\ldots$ & $\ldots$ & $\ldots$ & $\ldots$ & $\ldots$ & $\ldots$ & \\
\hline $3973.27 \ldots \ldots .$. & $\mathrm{Nd}$ II & 0.63 & 0.360 & $\ldots$ & 19.9 & $\ldots$ & $\ldots$ & 8.9 & $\ldots$ & $\ldots$ & $\ldots$ & 14.2 & $\ldots$ & $\ldots$ & $\ldots$ & $\ldots$ & $\ldots$ & $\ldots$ & $\ldots$ & $\ldots$ & $\ldots$ & 25.6 & $\ldots$ & 12.1 & 36.2 \\
\hline $4018.81 \ldots \ldots$ & $\mathrm{Nd}$ II & 0.06 & -0.850 & & 8.1 & $\ldots$ & $\ldots$ & Syn & $\ldots$ & $\ldots$ & $\ldots$ & $\ldots$ & 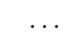 & & $\cdots$ & $\ldots$ & $\ldots$ & $\ldots$ & $\ldots$ & $\ldots$ & $\ldots$ & 11.2 & $\ldots$ & & 13.9 \\
\hline
\end{tabular}


TABLE 3-Continued

\begin{tabular}{|c|c|c|c|c|c|c|c|c|c|c|c|c|c|c|c|c|c|c|c|c|c|c|c|c|c|}
\hline \multirow{2}{*}{$\begin{array}{c}\text { WAVELENGTH } \\
(\AA)\end{array}$} & \multirow[b]{2}{*}{ Species } & \multirow{2}{*}{$\begin{array}{r}\text { L.E.P. } \\
(\mathrm{eV})\end{array}$} & \multirow[b]{2}{*}{$\log g f$} & \multicolumn{22}{|c|}{ Equivalent WIDTH $(\mathrm{m} \AA)^{\mathrm{a}}$} \\
\hline & & & & 1 & 2 & 3 & 4 & 5 & 6 & 7 & 8 & 9 & 10 & 11 & 12 & 13 & 14 & 15 & 16 & 17 & 18 & 19 & 20 & 21 & 22 \\
\hline $4021.34 \ldots \ldots$. & $\mathrm{Nd}$ II & 0.32 & -0.100 & $\ldots$ & 17.7 & $\ldots$ & 14.5 & 7.8 & $\ldots$ & 3.4 & $\ldots$ & 13.9 & $\ldots$ & $\ldots$ & $\ldots$ & $\ldots$ & & $\ldots$ & $\ldots$ & $\ldots$ & $\ldots$ & 26.8 & $\ldots$ & 7.2 & 35.1 \\
\hline $4061.09 \ldots \ldots$. & $\mathrm{Nd}$ II & 0.47 & 0.550 & $\ldots$ & 42.3 & $\ldots$ & 40.4 & 20.3 & 6.1 & 9.3 & 1.4 & 34 & $\ldots$ & $\ldots$ & $\ldots$ & $\ldots$ & $\ldots$ & $\ldots$ & $\ldots$ & $\ldots$ & $\ldots$ & 51.5 & $\ldots$ & 20 & 63.9 \\
\hline $4069.27 \ldots \ldots$ & $\mathrm{Nd}$ II & 0.06 & -0.570 & $\ldots$ & 13.6 & $\ldots$ & 15.6 & $\ldots$ & $\ldots$ & $\ldots$ & $\ldots$ & 9.9 & $\ldots$ & $\ldots$ & $\ldots$ & $\ldots$ & $\ldots$ & $\ldots$ & $\ldots$ & $\ldots$ & $\ldots$ & 22 & $\ldots$ & 9.1 & 34.3 \\
\hline $4109.46 \ldots \ldots$. & $\mathrm{Nd}$ II & 0.32 & 0.350 & $\ldots$ & 49.9 & $\ldots$ & 50.8 & 23.9 & $\ldots$ & 10.6 & $\ldots$ & 38.8 & $\ldots$ & $\ldots$ & $\ldots$ & $\ldots$ & $\ldots$ & $\ldots$ & $\ldots$ & $\ldots$ & $\ldots$ & 61.1 & $\ldots$ & 27.2 & 71.4 \\
\hline $4232.38 \ldots \ldots$. & $\mathrm{Nd}$ II & 0.06 & -0.470 & $\ldots$ & $\ldots$ & $\ldots$ & 24.1 & 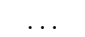 & $\ldots$ & 2.8 & $\ldots$ & $\ldots$ & $\ldots$ & $\ldots$ & $\ldots$ & $\ldots$ & & $\ldots$ & $\ldots$ & $\ldots$ & $\ldots$ & 24.8 & $\ldots$ & 10.9 & 37.4 \\
\hline $4446.39 \ldots \ldots$ & $\mathrm{Nd}$ II & 0.20 & -0.350 & $\ldots$ & 17 & $\ldots$ & 17.4 & 7.6 & $\ldots$ & 2.8 & $\ldots$ & 14.4 & $\ldots$ & $\ldots$ & $\ldots$ & $\ldots$ & $\ldots$ & $\ldots$ & $\ldots$ & $\ldots$ & $\ldots$ & 21.8 & $\ldots$ & 6 & 35.8 \\
\hline $4462.99 \ldots \ldots .$. & $\mathrm{Nd}$ II & 0.56 & -0.004 & $\ldots$ & 17.5 & $\ldots$ & 19.9 & 7.1 & $\ldots$ & 5 & $\ldots$ & 16.4 & $\ldots$ & $\ldots$ & $\ldots$ & $\ldots$ & $\ldots$ & $\ldots$ & $\ldots$ & $\ldots$ & $\ldots$ & 27.3 & $\ldots$ & 9.6 & 33.9 \\
\hline 3896.97....... & $\mathrm{Sm}$ II & 0.04 & -0.580 & $\ldots$ & 6.6 & $\ldots$ & 8.5 & 3.7 & $\ldots$ & $\ldots$ & $\ldots$ & 5.8 & $\ldots$ & $\ldots$ & $\ldots$ & $\ldots$ & & $\ldots$ & $\ldots$ & $\ldots$ & $\ldots$ & 10.5 & $\ldots$ & 3 & 23.9 \\
\hline $4318.94 \ldots \ldots$. & Sm II & 0.28 & -0.270 & $\ldots$ & 14.6 & $\ldots$ & 18.5 & 8.6 & $\ldots$ & 3 & $\ldots$ & 13.3 & $\ldots$ & $\ldots$ & $\ldots$ & $\ldots$ & $\ldots$ & $\ldots$ & $\ldots$ & $\ldots$ & $\ldots$ & 21.5 & $\ldots$ & 6.7 & 34 \\
\hline $4519.63 \ldots \ldots$ & Sm II & 0.54 & -0.430 & $\ldots$ & $\ldots$ & $\ldots$ & 8.2 & 4.1 & $\ldots$ & $\ldots$ & $\ldots$ & 6.1 & $\ldots$ & $\ldots$ & $\ldots$ & $\ldots$ & $\ldots$ & $\ldots$ & $\ldots$ & $\ldots$ & $\ldots$ & 11 & $\ldots$ & $\ldots$ & 20 \\
\hline $4577.69 \ldots \ldots$. & Sm II & 0.25 & -0.770 & $\ldots$ & 6.4 & $\ldots$ & 8.7 & 3.6 & $\ldots$ & 1.3 & $\ldots$ & 6.3 & $\ldots$ & $\ldots$ & $\ldots$ & $\ldots$ & $\ldots$ & $\ldots$ & $\ldots$ & $\ldots$ & $\ldots$ & 11.4 & $\ldots$ & $\ldots$ & 18.4 \\
\hline 3819.67........ & Eu II & 0.00 & 0.510 & Syn & Syn & $\ldots$ & Syn & Syn & $\ldots$ & Syn & $\ldots$ & Syn & $\ldots$ & $\ldots$ & $\ldots$ & $\ldots$ & $\ldots$ & $\ldots$ & $\ldots$ & $\ldots$ & $\ldots$ & Syn & Syn & Syn & $\ldots$ \\
\hline $4129.70 \ldots \ldots$ & Eu II & 0.00 & 0.220 & $\ldots$ & Syn & $\ldots$ & Syn & Syn & Syn & Syn & $\ldots$ & Syn & $\ldots$ & $\ldots$ & $\ldots$ & $\ldots$ & $\ldots$ & $\ldots$ & $\ldots$ & $\ldots$ & $\ldots$ & Syn & Syn & Syn & $\ldots$ \\
\hline $4205.05 \ldots \ldots$. & Eu II & 0.00 & 0.210 & Syn & Syn & $\ldots$ & Syn & Syn & $\ldots$ & Syn & $\ldots$ & Syn & $\ldots$ & $\ldots$ & $\ldots$ & $\ldots$ & $\ldots$ & $\ldots$ & $\ldots$ & $\ldots$ & $\ldots$ & Syn & Syn & Syn & \\
\hline $3768.40 \ldots \ldots$ & Gd II & 0.08 & 0.250 & $\ldots$ & 36.9 & $\ldots$ & 36.4 & 18.3 & 4.3 & 9.3 & $\ldots$ & 30.9 & $\ldots$ & $\ldots$ & $\ldots$ & $\ldots$ & $\ldots$ & $\ldots$ & $\ldots$ & $\ldots$ & $\ldots$ & 66 & $\ldots$ & 25.9 & 57.9 \\
\hline $3796.39 \ldots \ldots .$. & Gd II & 0.03 & 0.030 & $\ldots$ & $\ldots$ & $\ldots$ & $\ldots$ & 27.8 & $\ldots$ & $\ldots$ & $\ldots$ & $\cdots$ & $\ldots$ & $\ldots$ & $\ldots$ & $\ldots$ & $\ldots$ & $\ldots$ & $\ldots$ & $\ldots$ & $\ldots$ & Syn & $\ldots$ & 27.8 & \\
\hline 3844.58....... & Gd II & 0.14 & -0.510 & $\ldots$ & 15.2 & $\ldots$ & $\ldots$ & 5.9 & $\ldots$ & $\ldots$ & $\ldots$ & 10.5 & $\ldots$ & $\ldots$ & $\ldots$ & $\ldots$ & $\ldots$ & $\ldots$ & $\ldots$ & $\ldots$ & $\ldots$ & 19.5 & $\ldots$ & $\ldots$ & 32.2 \\
\hline $3702.86 \ldots \ldots$ & $\mathrm{Tb}$ II & 0.13 & 0.440 & $\ldots$ & Syn & $\ldots$ & 9.5 & $\ldots$ & $\ldots$ & $\ldots$ & $\ldots$ & 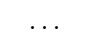 & $\ldots$ & $\ldots$ & $\ldots$ & $\ldots$ & $\ldots$ & $\ldots$ & $\ldots$ & $\ldots$ & & 26.3 & $\ldots$ & $\ldots$ & \\
\hline $3848.74 \ldots \ldots$ & $\mathrm{Tb}$ II & 0.00 & 0.280 & $\ldots$ & $\ldots$ & $\ldots$ & $\ldots$ & Syn & $\ldots$ & $\ldots$ & $\ldots$ & $\ldots$ & $\ldots$ & $\ldots$ & $\ldots$ & $\ldots$ & & $\ldots$ & $\ldots$ & $\ldots$ & $\ldots$ & $\ldots$ & $\ldots$ & $\ldots$ & Syn \\
\hline $4002.57 \ldots \ldots$. & $\mathrm{Tb}$ II & 0.64 & 0.100 & $\ldots$ & $\ldots$ & $\ldots$ & Syn & $\ldots$ & $\ldots$ & $\ldots$ & $\ldots$ & $\ldots$ & $\ldots$ & $\ldots$ & $\ldots$ & $\ldots$ & $\ldots$ & $\ldots$ & $\ldots$ & $\ldots$ & $\ldots$ & 6.7 & $\ldots$ & $\ldots$ & $\ldots$ \\
\hline $4005.47 \ldots \ldots$. & $\mathrm{Tb}$ II & 0.13 & -0.020 & $\cdots$ & $\ldots$ & $\ldots$ & $\ldots$ & $\ldots$ & $\ldots$ & $\ldots$ & $\ldots$ & $\ldots$ & $\ldots$ & $\ldots$ & $\ldots$ & $\ldots$ & $\ldots$ & $\ldots$ & $\ldots$ & $\ldots$ & $\ldots$ & 5.1 & $\ldots$ & $\ldots$ & Syn \\
\hline $3788.44 \ldots \ldots .$. & Dy II & 0.10 & -0.520 & $\ldots$ & 13 & $\ldots$ & $\ldots$ & 10.8 & $\ldots$ & 3.3 & $\ldots$ & 17.2 & $\ldots$ & $\ldots$ & $\ldots$ & $\ldots$ & $\ldots$ & $\ldots$ & $\ldots$ & $\ldots$ & $\ldots$ & 33.5 & $\ldots$ & 8.1 & 45.2 \\
\hline $3869.86 \ldots \ldots$ & Dy II & 0.00 & -0.940 & $\ldots$ & 10.5 & $\ldots$ & 12.5 & 5.6 & $\ldots$ & $\ldots$ & $\ldots$ & 10.1 & $\ldots$ & $\ldots$ & $\ldots$ & $\ldots$ & $\ldots$ & $\ldots$ & $\ldots$ & $\ldots$ & $\ldots$ & 25.7 & $\ldots$ & $\ldots$ & 32.9 \\
\hline $3996.69 \ldots \ldots$. & Dy II & 0.59 & -0.190 & $\ldots$ & 11.7 & $\ldots$ & 7.5 & 4.9 & $\ldots$ & $\ldots$ & $\ldots$ & 10.2 & $\ldots$ & $\ldots$ & $\ldots$ & $\ldots$ & $\ldots$ & $\ldots$ & $\ldots$ & $\ldots$ & $\ldots$ & 20.4 & $\ldots$ & $\ldots$ & 29.6 \\
\hline $4077.96 \ldots \ldots .$. & Dy II & 0.10 & -0.030 & $\ldots$ & Syn & $\ldots$ & Syn & Syn & . & & $\ldots$ & & $\ldots$ & $\ldots$ & $\ldots$ & $\ldots$ & $\ldots$ & $\ldots$ & $\ldots$ & $\ldots$ & & Syn & $\ldots$ & Syn & Syn \\
\hline $4103.31 \ldots \ldots .$. & Dy II & 0.10 & -0.370 & $\ldots$ & 31.3 & $\ldots$ & 40.8 & 18.1 & $\ldots$ & 5.8 & $\ldots$ & 24.9 & $\ldots$ & $\ldots$ & $\ldots$ & $\ldots$ & & $\ldots$ & $\ldots$ & $\ldots$ & $\ldots$ & 53.1 & $\ldots$ & 17.3 & 62.5 \\
\hline $3692.65 \ldots \ldots$. & Er II & 0.05 & 0.130 & $\ldots$ & 48.5 & $\ldots$ & 38 & 31.6 & $\ldots$ & $\ldots$ & $\ldots$ & 41.5 & $\ldots$ & $\ldots$ & $\ldots$ & $\ldots$ & $\ldots$ & $\ldots$ & $\ldots$ & $\ldots$ & $\ldots$ & 63.6 & $\ldots$ & 35.3 & 75.7 \\
\hline $3786.84 \ldots \ldots$ & Er II & 0.00 & -0.640 & $\ldots$ & Syn & $\ldots$ & $\ldots$ & Syn & $\ldots$ & $\ldots$ & $\ldots$ & Syn & $\ldots$ & $\ldots$ & $\ldots$ & $\ldots$ & $\ldots$ & $\ldots$ & $\ldots$ & $\ldots$ & $\ldots$ & Syn & $\ldots$ & $\ldots$ & Syn \\
\hline $3830.48 \ldots \ldots$ & Er II & 0.00 & -0.360 & $\ldots$ & $\ldots$ & $\ldots$ & $\ldots$ & $\ldots$ & $\ldots$ & $\ldots$ & $\ldots$ & $\ldots$ & $\ldots$ & $\ldots$ & $\ldots$ & $\ldots$ & $\ldots$ & $\ldots$ & $\ldots$ & $\ldots$ & $\ldots$ & 43.8 & $\ldots$ & 14.9 & $\ldots$ \\
\hline $3896.23 \ldots \ldots$. & Er II & 0.05 & -0.240 & $\ldots$ & $\ldots$ & $\ldots$ & Syn & Syn & $\ldots$ & $\ldots$ & $\ldots$ & Syn & $\ldots$ & $\ldots$ & $\ldots$ & $\ldots$ & & $\ldots$ & $\ldots$ & $\ldots$ & $\ldots$ & Syn & $\ldots$ & 27.5 & Syn \\
\hline $3938.63 \ldots \ldots$. & Er II & 0.00 & -0.520 & $\ldots$ & 17 & $\ldots$ & Syn & 9.9 & $\ldots$ & $\ldots$ & $\ldots$ & 10.1 & $\ldots$ & $\ldots$ & $\ldots$ & $\ldots$ & $\ldots$ & $\ldots$ & $\ldots$ & $\ldots$ & $\ldots$ & 29.2 & $\ldots$ & 7.1 & 31.2 \\
\hline $3700.26 \ldots \ldots$. & Tm II & 0.03 & -0.290 & $\ldots$ & $\ldots$ & $\ldots$ & $\ldots$ & $\ldots$ & $\ldots$ & $\ldots$ & $\ldots$ & $\ldots$ & $\ldots$ & $\ldots$ & $\ldots$ & $\ldots$ & $\ldots$ & $\ldots$ & $\ldots$ & $\ldots$ & $\ldots$ & 18.7 & $\ldots$ & $\ldots$ & 23 \\
\hline $3701.36 \ldots \ldots$ & Tm II & 0.00 & -0.420 & $\ldots$ & 6.4 & $\ldots$ & Syn & $\ldots$ & $\ldots$ & $\ldots$ & $\ldots$ & 4.3 & $\ldots$ & $\ldots$ & $\ldots$ & $\ldots$ & $\ldots$ & $\ldots$ & $\ldots$ & $\ldots$ & $\ldots$ & 13.9 & $\ldots$ & $\ldots$ & 13.6 \\
\hline $3795.76 \ldots \ldots$ & $\mathrm{Tm}$ II & 0.03 & -0.170 & $\ldots$ & 7.5 & $\ldots$ & 5.4 & 5.6 & $\ldots$ & $\ldots$ & $\ldots$ & 11.4 & $\ldots$ & $\ldots$ & $\ldots$ & $\ldots$ & $\ldots$ & $\ldots$ & $\ldots$ & $\ldots$ & $\ldots$ & 22.1 & $\ldots$ & 8.5 & 36.3 \\
\hline $3848.02 \ldots \ldots$. & $\mathrm{Tm}$ II & 0.00 & -0.130 & $\ldots$ & 10.9 & $\ldots$ & $\ldots$ & Syn & $\ldots$ & $\ldots$ & $\ldots$ & 10.7 & $\ldots$ & $\ldots$ & $\ldots$ & $\ldots$ & $\ldots$ & $\ldots$ & $\ldots$ & $\ldots$ & $\ldots$ & 22.2 & $\ldots$ & 9.1 & \\
\hline $3694.19 \ldots \ldots .$. & $\mathrm{Yb}$ II & 0.00 & -0.299 & $\ldots$ & Syn & $\ldots$ & Syn & Syn & $\ldots$ & $\ldots$ & $\ldots$ & Syn & $\ldots$ & $\ldots$ & $\ldots$ & $\ldots$ & $\ldots$ & $\ldots$ & $\ldots$ & $\ldots$ & $\ldots$ & Syn & $\ldots$ & Syn & Syn \\
\hline 4135.77........ & Os I & 0.52 & -1.260 & $\ldots$ & $\ldots$ & $\ldots$ & $\ldots$ & $\ldots$ & $\ldots$ & $\ldots$ & $\ldots$ & $\ldots$ & $\ldots$ & $\ldots$ & $\ldots$ & $\ldots$ & $\ldots$ & $\ldots$ & $\ldots$ & $\ldots$ & $\ldots$ & $\ldots$ & $\ldots$ & $\ldots$ & Syn \\
\hline $3513.65 \ldots \ldots$ & Ir I & 0.00 & -1.260 & $\ldots$ & Syn & $\ldots$ & $\ldots$ & Syn & $\ldots$ & $\ldots$ & $\ldots$ & $\ldots$ & $\ldots$ & $\ldots$ & $\ldots$ & $\ldots$ & $\ldots$ & $\ldots$ & $\ldots$ & $\ldots$ & $\ldots$ & $\ldots$ & $\ldots$ & $\ldots$ & $\ldots$ \\
\hline $4019.12 \ldots \ldots .$. & Th II & 0.00 & -0.270 & $\ldots$ & Syn & $\ldots$ & Syn & Syn & $\ldots$ & $\ldots$ & $\ldots$ & Syn & $\ldots$ & $\ldots$ & $\ldots$ & $\ldots$ & $\ldots$ & $\ldots$ & $\ldots$ & $\ldots$ & $\ldots$ & Syn & $\ldots$ & Syn & Syn \\
\hline
\end{tabular}

Noте.-Table 3 is also available in machine-readable form in the electronic edition of the Astrophysical Journal Supplement.

"Identification of objects is given in Table 1. "Syn" denote that the abundance derived by spectral synthesis technique. 
Bonifacio, Monai, \& Beers 2000; Allende Prieto et al. 2000). For the majority of these stars, no elemental abundance results based on high-resolution spectroscopy has been previously obtained. Due to the selection criteria employed, it should be noted that our sample emphasizes stars that are either definite, or suspected, $r$-process-enhanced, metal-poor stars, which will impact the discussion of the distribution of the observed abundances of neutron-capture elements for these stars presented in Honda et al. (2004, hereafter Paper II).

Since our primary purpose is to investigate the neutroncapture elements, we selected giants, whose metal lines are generally stronger than metal-poor dwarfs near the mainsequence turnoff due to their lower effective temperatures. Exceptions are HD 140283 and BS 17583-100, which were observed for comparison purposes. A rather large fraction of very metal-poor stars exhibit enhancements of carbon (e.g., Beers et al. 1992; Rossi, Beers, \& Sneden 1999), up to $25 \%$ by some recent estimates. However, strongly carbon-enhanced stars $([\mathrm{C} / \mathrm{Fe}] \gtrsim+1.0)$ are excluded from our sample, because contamination arising from molecular lines $(\mathrm{CH}$ and $\mathrm{CN})$ makes the analysis of lines of neutron-capture elements difficult and causes particular problems with regard to features of Th and U. An exception is the star CS 22892-052, which is known to exhibit an extremely large excess of $r$-process elements, and a large carbon enhancement, on the order of $[\mathrm{C} / \mathrm{Fe}] \approx+1.1$ (see Norris et al. 1997 for a discussion of the impact on studies of Th in such stars).

The 22 stars selected for our program are listed in Table 1. In this table we also list the apparent $V$ magnitudes and $B-V$ colors, taken from the list of T. C. Beers et al. (2004, in preparation) and the SIMBAD database. As can be seen, most of our targets fall in the range $0.7 \leq B-V \leq 1.2$ and are likely to be giant-branch stars.

\subsection{Subaru/HDS Observations}

High-resolution spectra of our program stars were obtained during the commissioning phase of HDS between 2000 July and 2001 July - a detailed log is provided in Tables 1 and 2. The HDS detector is a mosaic system of two EEV-CCDs, each with $2048 \times 4100$ pixels. HDS is designed to achieve high spectral resolving power, high sensitivity, and (almost) complete wavelength in the blue region. These are essential characteristics for our program, since the weak absorption lines of neutron-capture elements fall primarily in the near UV-blue range. Details of the design of the spectrograph and its performance are provided by Noguchi et al. (2002).

For the observations reported herein, the slit width of the spectrograph was set to $0.4(200 \mu \mathrm{m})$ or $0.72(360 \mu \mathrm{m})$, which corresponds to a spectral resolving power of $R \simeq 90,000$ or 50,000 , respectively (Table 1). An exception is HD 186478 , which was observed with a $0.36(180 \mu \mathrm{m})$ slit. The high resolving power and oversampling of the spectra (roughly six pixels per resolution element for 0 "'72 slit width) obtained by HDS are particularly valuable for the study of lines affected by hyperfine splitting and isotope shifts and/or from blending with other atomic and molecular lines. Our observations covered the wavelength from $3500 \AA$ (3400 $\AA$ for a few objects) to $5200 \AA$ ( $5100 \AA$ for a few stars), with a lack of data between 4350 and $4400 \AA$ ( 4230 and $4280 \AA$ ) due to the gap between the two CCDs.

Since our observations were made in the early phase of HDS commissioning, there were some limitations of various components in the preslit unit, especially the image rotators and the atmospheric differential dispersion corrector (ADC). The ADC was installed at the end of 2000 and has been applied from the 2001 January run forward. An image rotator was needed for target acquisition and guiding in the observing run conducted in 2000. In the 2000 July run, the blue spectra of HD 115444, HD 122563, and HD 140283 were obtained using the image rotator optimized for the red, as the blue one was unavailable at that time. In addition, the slit was fixed to the north/south direction, instead of being aligned along the parallactic angle, due to limitations in guiding. For this reason, significant light loss occurred in the short-wavelength region where the effect of atmospheric differential dispersion is quite large, degrading the spectral quality at the shortest wavelengths. We note that these three objects were reobserved in later observing runs so that sufficient quality could be achieved.

In most cases, the spectra of fainter stars in our program were obtained by combining several $1800 \mathrm{~s}$ exposures. This choice was motivated by the desire to limit the degradation of the spectra due to cosmic-ray events. The total exposure time for each object ranges from $900 \mathrm{~s}$ (for the brightest star) to $9751 \mathrm{~s}$ (for the faintest star). The signal-to-noise ratios at $\sim 4000 \AA$ are $40<\mathrm{S} / \mathrm{N}<450$ per pixel $(100<\mathrm{S} / \mathrm{N}<900$ per resolution element), as shown in Table 1 . There was no need, in general, for the use of on-chip CCD binning, because the read-out noise was not an important source of noise in this study. The exception is CS $31082-001$, for which $2 \times 2$ binning mode was used, because this object was observed during another observing program in which the binning mode was employed.

For reduction of the spectral data, we obtained bias frames, halogen lamp frames for flat-fielding, and Th-Ar spectra for wavelength calibration. Though dark frames were obtained in each run to check the dark current of the CCD, it turned out to be very small; hence, no dark correction was made during data reduction.

The echelle data were processed using the IRAF ${ }^{14}$ software package in a standard manner. Here we summarize the flow of the data reduction. We first corrected the fluctuation of the bias level by subtracting the average of the counts in the overscan region from each frame. The median of the bias frame was then subtracted from all frames. We then divided the object frames by the average of the flat-field frames. The scattered light level was estimated by obtaining surface fits of the interorder regions and then was subtracted from each object frame. One-dimensional spectra were extracted after removing cosmic-ray events. Since the wavelength ranges covered by the CCD are much wider than the free spectral range in the near UV-blue region, we trimmed the spectral orders when the count level fell below a useful level. In practice, this meant that roughly 1000 pixels were trimmed from the blue portions of each order, and 500 pixels were trimmed from the red portions of each order, respectively.

The $\mathrm{S} / \mathrm{N}$ ratios of the spectra were evaluated from the peak count in echelle order $149(\sim 4000 \AA)$. The $\mathrm{S} / \mathrm{N}$ ratios per pixel $(0.012 \AA)$ and per resolution element are given in Table 1. It should be noted that the sampling rate of HDS is quite high in the $R \simeq 50,000$ spectra (six pixels per resolution element); hence, the $\mathrm{S} / \mathrm{N}$ ratios per pixel may seem rather low in some cases.

\footnotetext{
${ }^{14}$ IRAF is distributed by National Optical Astronomy Observatory, which is operated by the Association of Universities for Research in Astronomy, Inc., under cooperative agreement with the National Science Foundation.
} 
Sample spectra for nine of our program stars in the regions near $4000 \AA$ (which includes the Th II $4019 \AA$ line) and $4100 \AA$ (which includes the Eu II $4129 \AA$ line) are shown in Figures 1 and 2, respectively. HD 140283 and HD 122563 are familiar, well-studied metal-poor stars. BS $16920-017$ is the star with the lowest metallicity in our study, with $[\mathrm{Fe} / \mathrm{H}]=-3.1$. CS 22952-015 has been studied by previous authors (McWilliam et al. 1995a; Ryan et al. 1996). In the spectrum of CS 22183-031, the Eu II $4129 \AA$ feature is detected, but it is quite weak. Given the paucity of information on the $\mathrm{Eu}$ abundances of stars with $[\mathrm{Fe} / \mathrm{H}] \sim-3.0$, this object should be reobserved at higher $\mathrm{S} / \mathrm{N}$ in order to obtain a better measurement. The other four objects in these figures show enhancements of the $r$-process elements. CS 22892-052 and CS 31082-001 are well-known, extremely $r$-processenhanced, very metal-poor giants (Sneden et al. 1996; Cayrel et al. 2001). These two objects clearly show the Th II $4019 \AA$ line, as well as very strong Eu II $4129 \AA$ features. HD 115444 is the object shown by Westin et al. (2000) to exhibit a moderate excess of $r$-process elements. CS 30306-132 turned out to exhibit excesses of the neutron-capture elements, as discovered during the present work. The Th II 4019 Å line was clearly detected in this object (see Paper II for details).

\section{EQUIVALENT WIDTH MEASUREMENTS}

We identified Fe absorption lines between 3700 and $5100 \AA$, which will be used to determine the atmospheric parameters for the abundance analysis using model atmospheres. Identification of these lines was mostly made on the basis of the line list provided by Westin et al. (2000). Equivalent widths were measured for clear, unblended lines of $\mathrm{Fe}$ I and Fe II by fitting Gaussian profiles to the observations using the spectral analysis software SPTOOL, developed by Y. Takeda (2003, private communication). We excluded lines from our analysis that may be significantly blended with other absorption lines. The blending with other atomic lines was checked by using the atomic line list by Kurucz \& Bell (1995).

Gaussian fitting may not reproduce well the wings of strong lines. However, for weak lines, the difference in derived equivalent widths from evaluations based on Gaussian fitting and those based on direct integration over the observed line profile is very small. Since our analysis relies exclusively on weak lines, we measured all equivalent widths by the Gaussian fitting procedure.

In addition to $\mathrm{Fe}$ lines, we also identified absorption lines of other elements, using the line lists provided by Westin et al. (2000) and Sneden et al. (1996). For Ba lines, we adopt the list of McWilliam (1998). For $\mathrm{La}, \mathrm{Eu}$, and Tb lines, we take from the list of Lawler et al. (Lawler, Bonvallet, \& Sneden 2001a; Lawler et al. 2001b, 2001c). We use the newest data also for $\mathrm{Nd}$ and $\mathrm{Yb}$, which are derived by Den Hartog et al. (2003) and C. Sneden (2003, private communication). Equivalent widths of these lines were also measured in the same manner as applied to the Fe lines. The measured equivalent widths are given in Table 3. The line data (lower excitation potentials, LEP, and the $g f$-values) are also listed in Table 3.

\subsection{Estimates of Internal Errors}

We estimate the random (internal) errors of our derived line strengths by determining the differences in measured equivalent widths from two measurements of each spectrum obtained with different individual exposures.
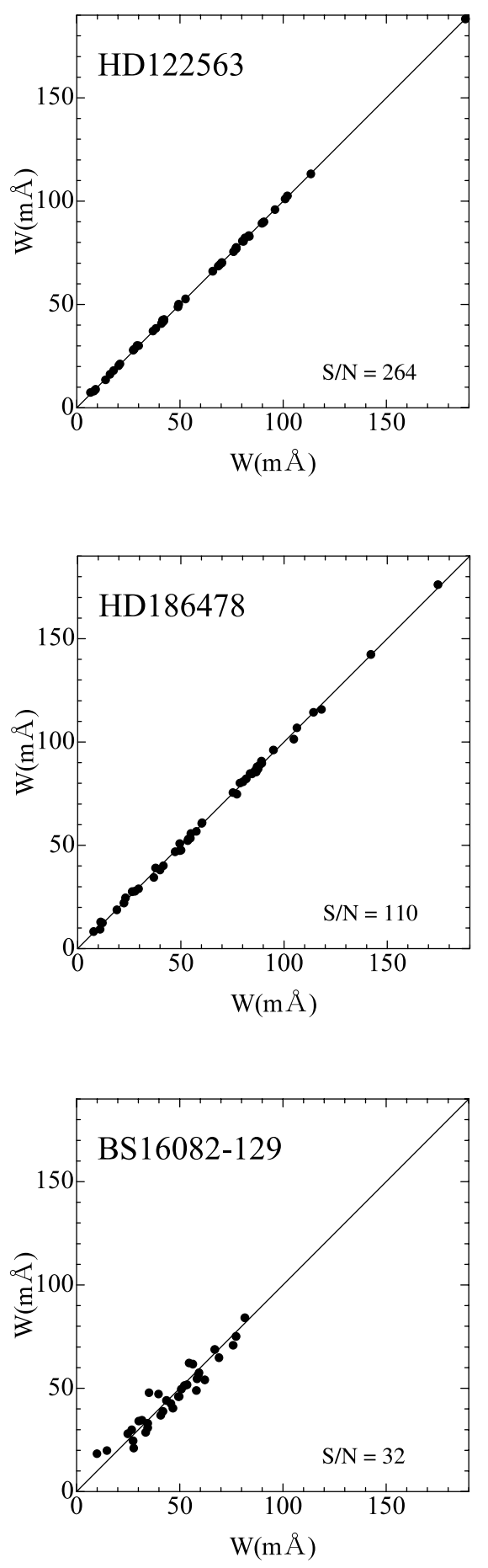

FIG. 3.-Comparison of equivalent width $(W)$ measurements for spectra obtained by individual HDS exposures for HD 122563 (top), HD 186478 (middle), and BS 16082-129 (bottom). The $\mathrm{S} / \mathrm{N}$ ratio of the final summed spectra are given in the individual panels. See text for details.

We selected three objects, HD 122563, HD 186478, and BS $16082-129$, as representative of stars observed with high, moderate, and rather low $\mathrm{S} / \mathrm{N}$ ratios, respectively. The number of photons collected by each exposure is about 70,000, 12,000, and 1000 at $4000 \AA$ for HD 122563, HD 186478, and BS 16082-129, respectively. Comparisons between the two measurements of weak Fe lines for these three objects are shown in Figure 3. No systematic differences between the individual measurements are evident. The standard deviations 

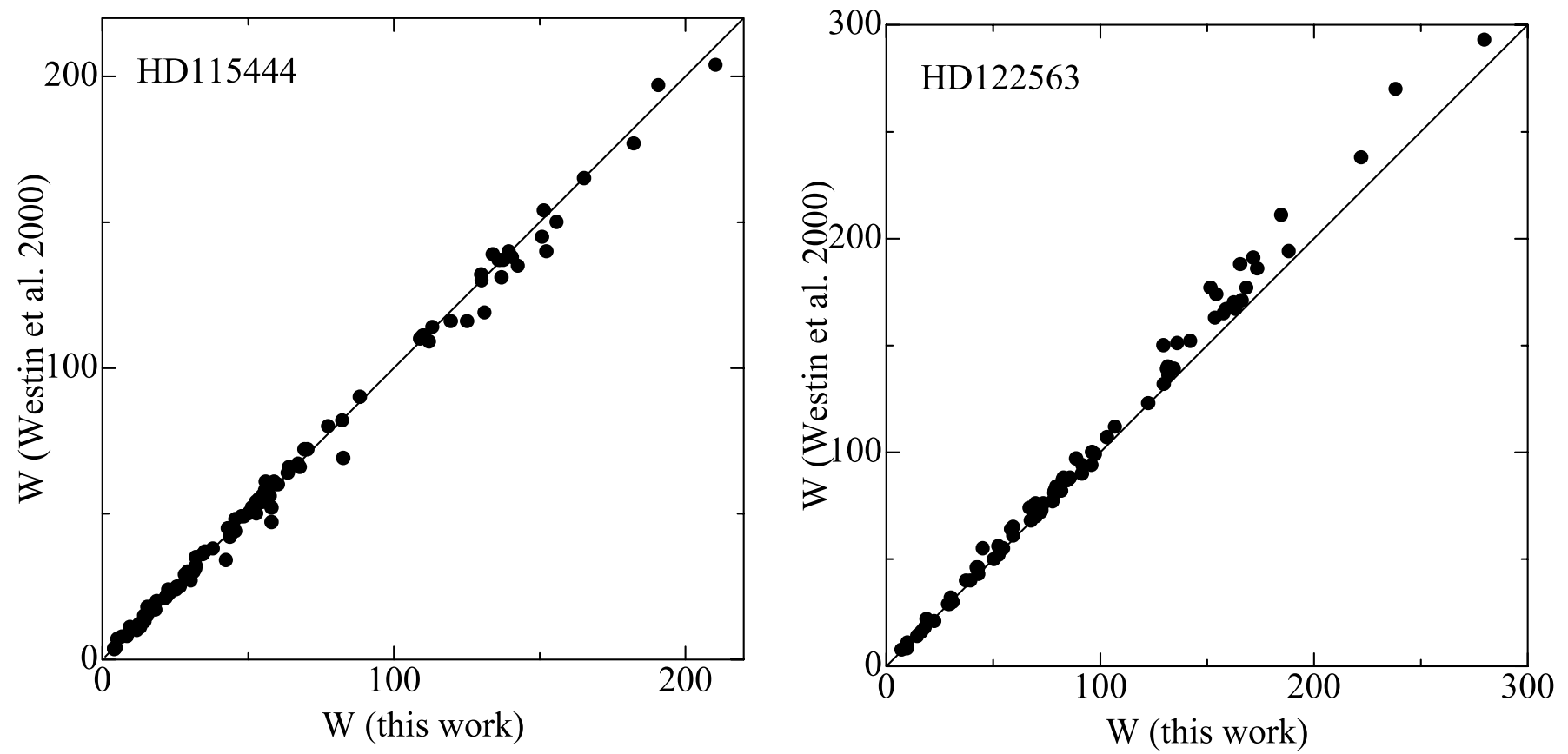

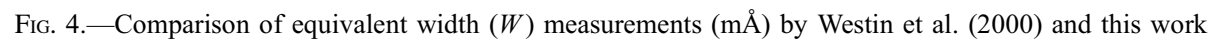
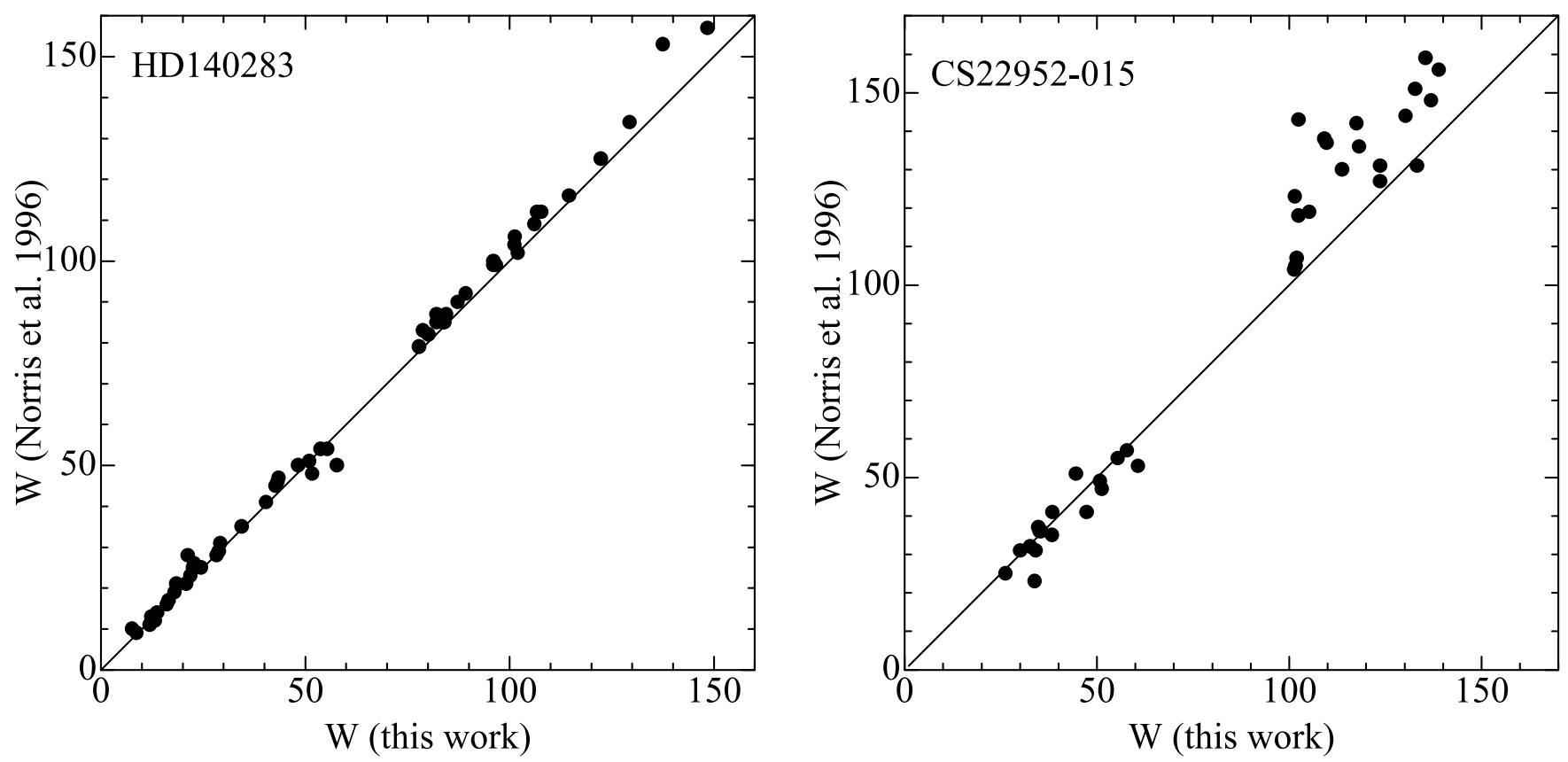

FIG. 5.-Comparison of equivalent width $(W)$ measurements $(m \AA)$ by Norris et al. (1996) and this work 

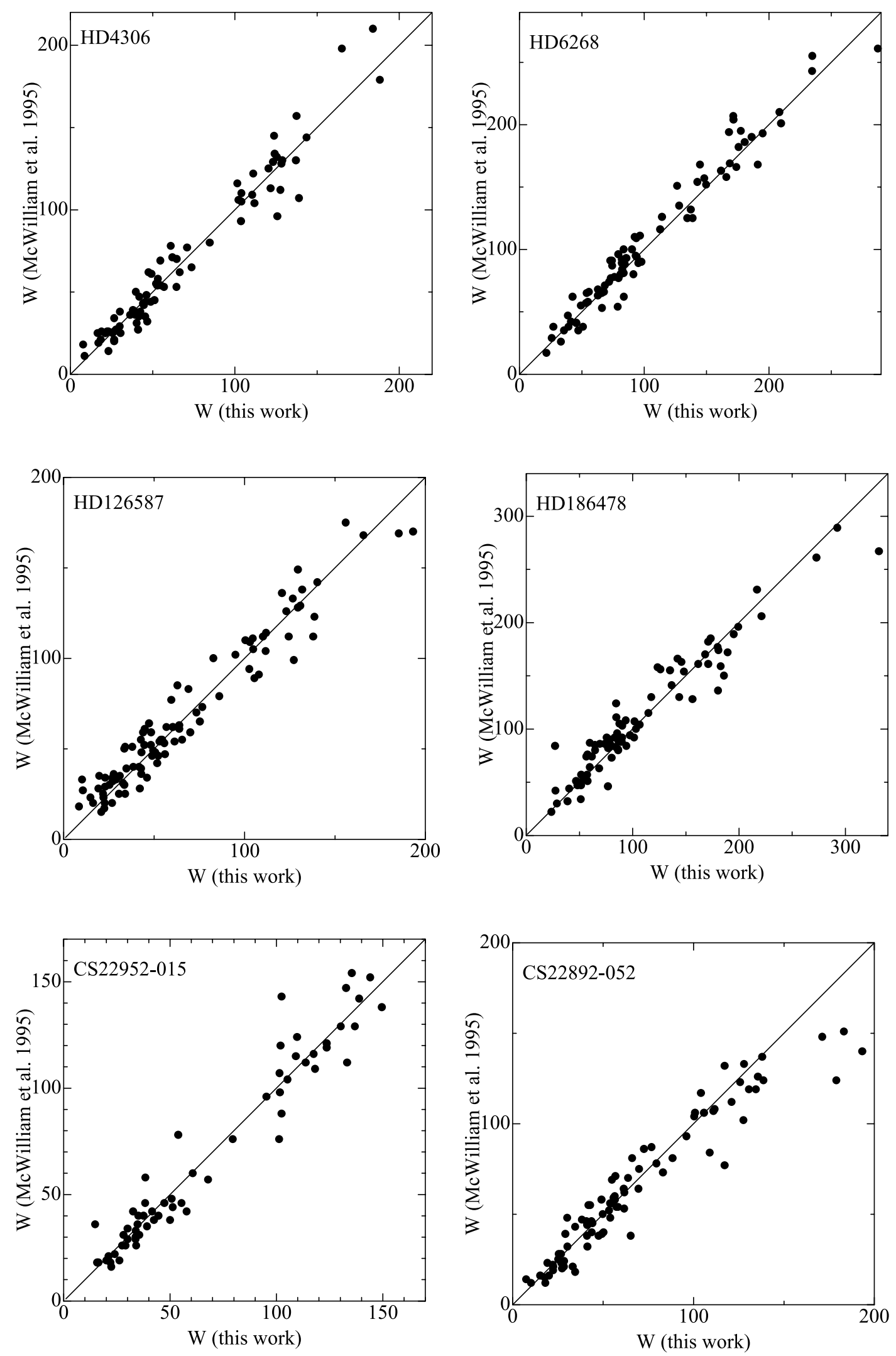

FIG. 6. - Comparison of equivalent width $(W)$ measurements (mÅ) by McWilliam et al. (1995b) and this work 


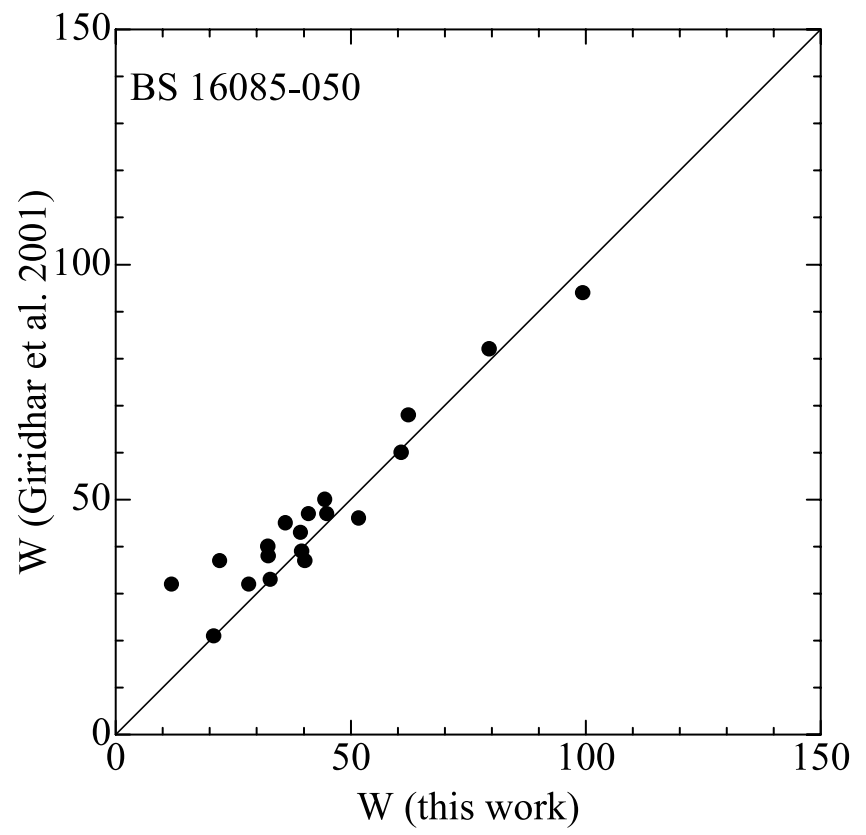

FIG. 7.-Comparison of equivalent width $(W)$ measurements (m $\AA$ ) by Giridhar et al. (2001) and this work.

in the differences of the two measurements for HD 122563, HD 186478, and BS $16082-129$ are $0.39,1.26$, and $4.98 \mathrm{~m} \AA$, respectively.

The uncertainly in the measured equivalent widths may also be roughly estimated, based on the $\mathrm{S} / \mathrm{N}$ ratio of the spectrum, as (line width) $\times(\mathrm{S} / \mathrm{N})^{-1}$. The typical line widths for giant stars in our sample is $7.5 \mathrm{~km} \mathrm{~s}^{-1}(100 \mathrm{~m} \AA$ at $4000 \AA)$. The uncertainty expected from the $\mathrm{S} / \mathrm{N}$ ratio of each spectrum, which is taken to be the square root of the number of detected photons, is $0.38,0.90$, and $3.2 \mathrm{~m} \AA$ for HD 122563, HD 186478, and BS 16082-129, respectively. The random errors measured above show a reasonable agreement with these values, though the measured ones are slightly higher than those predicted from the $\mathrm{S} / \mathrm{N}$ ratios. This small discrepancy may arise because the $\mathrm{Fe}$ lines used in our analysis are randomly distributed across individual orders, which have a blaze function variation in their $\mathrm{S} / \mathrm{N}$ levels, while the number of photons was measured at the center of the echelle blaze profile.

\subsection{Comparisons with Previous Studies}

Several stars in our sample have also been investigated by previous authors conducting high-resolution abundance studies. In Figures 4, 5, 6, 7, and 8, the equivalent widths estimated from the present data are compared with those reported by others.

Westin et al. (2000) analyzed high-resolution $(R \sim 60,000)$ and high $\mathrm{S} / \mathrm{N}(\sim 200$ at $4000 \AA)$ spectra of the two bright objects HD 122563 and HD 115444, obtained with the " $2 \mathrm{~d}$ coude" cross-dispersed echelle spectrograph at the McDonald Observatory $2.7 \mathrm{~m}$ telescope; their results are compared with ours in Figure 4. An excellent agreement is found, with a very small scatter $(\sim 2 \%)$ in the range of equivalent width less than $150 \mathrm{~m} \AA$. There is a small $(\sim 10 \%)$ difference in the range of equivalent widths larger than $150 \mathrm{~m} \AA$ for HD 122563. The reason for this difference is not clear, but this difference does not have a significant influence on the abundance analysis because we are primarily concerned with weak lines.
Norris, Ryan, \& Beers (1996) obtained high-resolution spectra for two of the very metal-poor stars included in our sample, using the coudé spectrograph (UCLES) at the AngloAustralian Telescope. They studied spectra with $R \sim 40,000$ of CS 22952-015 (S/N 50) and HD $140283(\mathrm{~S} / \mathrm{N} \sim 200)$. In Figure 5, the equivalent widths measured for our spectra are compared with theirs for these two objects. The agreement is very good for HD 140283; there is no systematic difference, and the dispersion is quite small $(\sim 2 \%)$. On the other hand, the scatter in the comparison for CS 22952-015 is larger, and our equivalent widths are systematically smaller than those of Norris et al. (1996) $(\sim 10 \%)$ for lines with large equivalent widths ( $>100 \mathrm{~m} \AA$ ). The large scatter is presumably due to the lower $\mathrm{S} / \mathrm{N}$ ratios in the CS 22952-015 spectra than those of HD 140283 in both studies. Most of the lines with equivalent widths stronger than $100 \mathrm{~m} \AA$ come from the portions of the spectra at wavelengths blueward of $4000 \AA$. We suspect that the discrepancy found for these strong lines is due to errors in the measurements by Norris et al. (1996), which were based on spectra of rather low $\mathrm{S} / \mathrm{N}$ in this wavelength region.

In Figure 6, equivalent widths for HD 4306, HD 6268, HD 126587, HD 186478, CS 22952-015, and CS 22892-052, reported by McWilliam et al. (1995b), are compared with ours. Their spectra were obtained with the "2D-FRUTTI" photoncounting imager at the Las Campanas $2.5 \mathrm{~m}$ telescope. The typical S/N of the observations obtained by McWilliam et al. (1995b) is $\mathrm{S} / \mathrm{N} \sim 40$ with $R \sim 22,000$ at $4800 \AA$. Their wavelength coverage extends from 3600 to $7600 \AA$. In spite of the large dispersion in the equivalent widths between these two set of measurements, which is surely due to the low $\mathrm{S} / \mathrm{N}$ ratios in the spectra of McWilliam et al. (1995b), the equivalent widths of McWilliam et al. (1995b) exhibit no systematic difference with respect to ours. One exception is the comparison with four strong lines in CS 22892-052. We suspect that this deviation is due to errors in the data of McWilliam et al. (1995b), because these lines exist in the shortest wavelength regions, where the quality of the McWilliam et al. data is quite low.

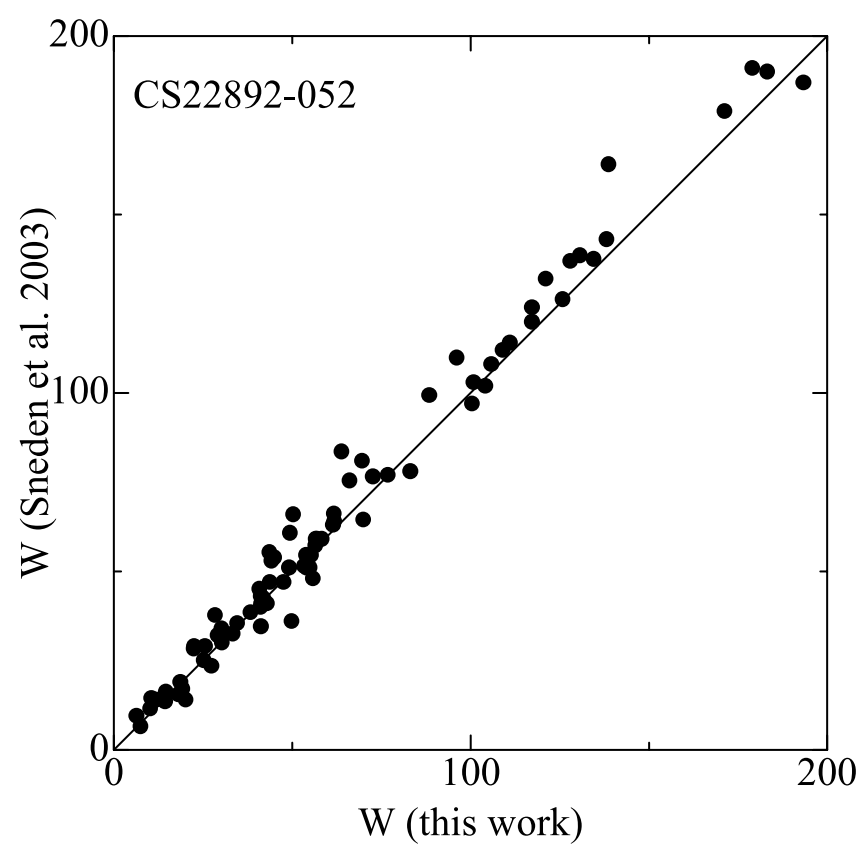

FIG. 8.-Comparison of equivalent width $(W)$ measurements (m $\AA$ ) by Sneden et al. (2003) and this work. 
TABLE 4

Radial Velocities Compared with Previous Studies

\begin{tabular}{|c|c|c|c|}
\hline Number & Object & Radial Velocity & References \\
\hline 1 & HD 4306 & $-69.69 \pm 0.29$ & This work (2000 Aug 19) \\
\hline $1 \ldots \ldots \ldots \ldots \ldots \ldots \ldots \ldots$ & HD 4306 & $-63.3 \pm 0.2$ & McWilliam et al. $1995 \mathrm{~b}$ \\
\hline $2 \ldots \ldots \ldots \ldots \ldots \ldots \ldots \ldots \ldots$ & HD 6268 & $39.20 \pm 0.27$ & This work (2000 Aug 18) \\
\hline $2 \ldots \ldots \ldots \ldots \ldots \ldots \ldots \ldots$ & HD 6268 & $38.9 \pm 0.3$ & McWilliam et al. 1995b \\
\hline 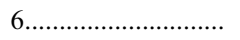 & HD 122563 & $-27.20 \pm 0.33$ & This work (2000 Jul 4) \\
\hline 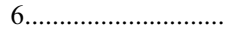 & HD 122563 & $-26.52 \pm 0.34$ & This work (2001 Jan 29) \\
\hline 6............................. & HD 122563 & $-27.0 \pm 0.34$ & Norris et al. 1996 \\
\hline 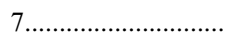 & HD 126587 & $148.72 \pm 0.72$ & This work (2001 Jan 27 Jan) \\
\hline 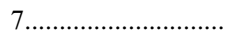 & HD 126587 & $149.10 \pm 0.24$ & This work (2001 Jan 31) \\
\hline 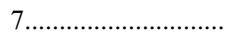 & HD 126587 & $150.0 \pm 0.3$ & McWilliam et al. $1995 \mathrm{~b}$ \\
\hline 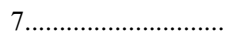 & HD 126587 & $149.40 \pm 0.17$ & Carney et al. 2003 \\
\hline 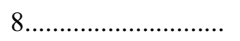 & HD 140283 & $-171.17 \pm 0.29$ & This work (2000 Jul 4) \\
\hline 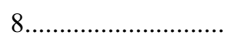 & HD 140283 & $-170.23 \pm 0.19$ & This work (2000 Aug 17) \\
\hline $8 \ldots \ldots \ldots \ldots \ldots \ldots \ldots \ldots \ldots$ & HD 140283 & $-171.9 \pm 0.19$ & Norris et al. 1996 \\
\hline 9 & HD 186478 & $30.52 \pm 0.31$ & This work (2000 Aug 20) \\
\hline 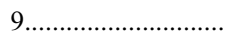 & HD 186478 & $32.7 \pm 0.3$ & McWilliam et al. $1995 \mathrm{~b}$ \\
\hline 9 & HD 186478 & $30.43 \pm 0.12 *$ & Carney et al. 2003 \\
\hline $19 \ldots \ldots \ldots \ldots \ldots \ldots \ldots$ & CS 22892-052 & $12.72 \pm 0.49$ & This work (2001 Jul 22) \\
\hline 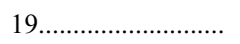 & CS 22892-052 & $13.1 \pm 0.3$ & McWilliam et al. $1995 \mathrm{~b}$ \\
\hline $19 \ldots \ldots \ldots \ldots \ldots \ldots \ldots \ldots$ & CS $22892-052$ & $13.6 \pm 0.3$ & Norris et al. 1996 \\
\hline 19 & CS $22892-052$ & $12.48 \pm 0.61^{*}$ & Preston \& Sneden 2001 \\
\hline $20 \ldots \ldots \ldots \ldots \ldots \ldots \ldots \ldots \ldots$ & CS 22952-015 & $-20.07 \pm 0.71$ & This work (2000 Nov 11$)$ \\
\hline $20 \ldots \ldots \ldots \ldots \ldots \ldots \ldots \ldots \ldots$ & CS 22952-015 & $-18.8 \pm 0.4$ & McWilliam et al. $1995 \mathrm{~b}$ \\
\hline $20 \ldots \ldots \ldots \ldots \ldots \ldots \ldots \ldots$ & CS 22952-015 & $-19.2 \pm 1$ & Norris et al. 1996 \\
\hline $22 \ldots \ldots \ldots \ldots \ldots \ldots \ldots$ & CS 31082-001 & $138.91 \pm 0.30$ & This work (2001 Jul 30) \\
\hline $22 \ldots \ldots \ldots \ldots \ldots \ldots \ldots \ldots$ & CS 31082-001 & $139.05 \pm 0.05$ & Hill et al. 2002 \\
\hline
\end{tabular}

Note.-Asterisks $(*)$ denote the mean value of their measurements.

In Figure 7, equivalent widths for BS 16085-050, reported by Giridhar et al. (2001), are compared with ours. Their spectra were obtained with the Apache Point Observatory's $3.5 \mathrm{~m}$ telescope and vacuum-sealed echelle spectrograph. Though the comparison shows a rather large dispersion, which is likely due to the low $\mathrm{S} / \mathrm{N}$ of the spectrum reported by Giridhar et al. (2001), there is no systematic difference between the two measurements. Although they also reported the equivalent widths of CS 22169-035, there are only four iron lines that were observed by both studies. Therefore, we do not show the comparison for this object.

Recently, Sneden et al. (2003) reported the results of a new detailed analysis of CS 22892-052. Their optical spectrum was obtained with Keck I/HIRES, McDonald $2.7 \mathrm{~m} / 2 \mathrm{~d}$-coudé, and VLT/UVES. In Figure 8, we compare our measured equivalent widths for CS 22892-052 with theirs. There exists no systematic difference between the two measurements, and the agreement is better than that found in the comparison with McWilliam et al. (1995b) for the same star.

\section{MEASUREMENTS OF RADIAL VELOCITIES}

Information on radial velocities and (where proper motions are available) on the space motion of stars of the halo is required to understand the structure and formation of the Galaxy. Precise radial velocity measurements are of particular importance for the moderately and highly $r$-process-enhanced, metal-poor stars, in order to check on their possible binarity, as this may impact the likely astrophysical site(s) of the $r$-process. For example, Qian \& Wasserburg (2001) have suggested that the $r$-process-enhanced, metal-poor stars were produced by contamination from companions that underwent Type II SN explosions.

Measurements of radial velocities were made for selected clean iron lines used in the equivalent width measurements. The wavelengths of the lines were measured, then compared with the rest (laboratory) values. For the objects observed at two different epochs, measurements were obtained for each spectrum. The measured heliocentric radial velocities, and their estimated standard deviations, are listed in Table 2.

Measurements of radial velocities have been previously obtained by a number of authors for several stars in our sample. However, most of them were based on low- or mediumresolution spectroscopy (e.g., Bond 1980; Norris, Bessell, \& Pickles 1985). We choose to compare our measurements with only the results of high-dispersion spectroscopy from recent studies. The comparisons are given in Table 4. No significant variation in radial velocity is found for most objects. HD 4306 and HD 186478 show changes of radial velocity of about 6 and $2 \mathrm{~km} \mathrm{~s}^{-1}$, respectively, suggesting that both stars may be members of binary systems. Indeed, Carney et al. (2003) have 
obtained an orbital solution for HD 186478, showing it to be a long-period binary $(P \approx 550$ days $)$ with a low amplitude, on the order of $3 \mathrm{~km} \mathrm{~s}^{-1}$. No definitive conclusion can yet be achieved for HD 4306; hence, further radial velocity monitoring is required to confirm its possible binarity. We note that HD 186478 is a moderately $r$-process-enhanced star, with $[\mathrm{Eu} / \mathrm{Fe}] \approx+0.5$ (Johnson \& Bolte 2001). Preston \& Sneden (2001) investigated the variations of radial velocity for the extremely $r$-processenhanced star CS 22892-052 in detail. However, there is still no clear evidence of binarity, as the suspected amplitude of the variation is quite small. We did not find evidence of binarity for any of our other $r$-process-enhanced stars.

\section{SUMMARY}

We have obtained high-resolution, high-S/N ratio, spectra for 22 very metal-poor stars with Subaru/HDS, taken during the commissioning phase of this instrument. These stars were selected so as to include as many objects with known (or suspected) enhancement of $r$-process elements as possible. In this paper we have reported the measurements of equivalent widths for isolated absorption lines in the reduced spectra, and also precision radial velocities (in some cases, at several epochs), for each star. Comparisons of our measured equivalent widths with previous work demonstrates that there exists no systematic differences, except in the cases of stronger lines in a few objects for which the $\mathrm{S} / \mathrm{N}$ ratios of the previous work was rather low. In the following papers of this series (Paper II and others in preparation), the results of the detailed abundance analysis for these data will be presented.

We thank all of staff members of the Subaru telescope, NAOJ, for their help during the observations. T. C. B. acknowledges partial support from grants AST 00-98508 and AST 00-98548 awarded by the US National Science Foundation. Most of the data reduction was carried out at the Astronomical Data Analysis Center (ADAC) of the National Astronomical Observatory of Japan.
Allende Prieto, C., Rebolo, R., García López, R, Serra-Ricart, M., Beers, T. C., Rossi, S., Bonifacio, P., \& Molaro, P. 2000, AJ, 120, 1516

Aoki, W., Norris, J. E., Ryan, S. G., Beers, T. C., Ando, H., \& Tsangarides, S. 2002, ApJ, 580, 1149

Beers, T. C. 1999 , Ap\&SS, 265, 547

Beers, T. C., Preston, G. W., \& Shectman, S. A. 1985, AJ, 90, 2089 1992, AJ, 103, 1987

Bond, H. E. 1980, ApJS, 44, 517

Bonifacio, P., Monai, S., \& Beers, T. C. 2000, AJ, 120, 2065

Burris, D. L., Pilachowski, C. A., Armandroff, T. E., Sneden, C., Cowan, J. J., \& Roe, H. 2000, ApJ, 544, 302

Carney, B. W., Latham, D. W., Stefanik, R. P., Laird, J. B., \& Morse, J. A. 2003, AJ, 125, 293

Carretta, E., Gratton, R. G., Cohen, J. G., Beers, T. C., \& Christlieb, N. 2002, AJ, 124, 481

Cayrel, R., et al. 2001, Nature, 409, 691 2004, A\&A, in press

Christlieb, N. 2003, Rev. Mod. Astron., 16, 191

Christlieb, N., \& Beers, T. C. 2000, Subaru HDS Workshop on Stars and Galaxies: Decipherment of Cosmic History with Spectroscopy (Tokyo: NAOJ)

Christlieb, N., Wisotzki, L., Reimers, D., Homeier, D., Koester, D., \& Heber, U. 2001, A\&A, 366, 898

Christlieb, N., et al. 2002, Nature, 419, 904

Cowan, J. J., et al. 2002, ApJ, 572, 861

Den Hartog, E. A., Lawler, J. E., Sneden, C., \& Cowan, J. J. 2003, ApJS, 148,543

Fields, B. D., Truran, J. W., \& Cowan, J. J. 2002, ApJ, 575, 845

Gallino, R., Arlandini, C., Busso, M., Lugaro, M., Travaglio, C., Straniero, O., Chieffi, A., \& Limongi, M. 1998, ApJ, 497, 388

Giridhar, S., Lambert, D. L., Gonzales, G., \& Pandey, G. 2001, PASP, 113, 519

Hill, V., et al. 2002, A\&A, 387, 560

Honda, S., Aoki, W., Kajino, T., Ando, H., Beers, T. C., Izumiura, H., Sadakane, K., \& Takada-Hidai, M. 2004, ApJ, in press (Paper II)

Ishimaru, Y., \& Wanajo, S. 1999, ApJ, 511, L33

Johnson, J. A., \& Bolte, M. 2001, ApJ, 554, 888

Kurucz, R. L., \& Bell, B. 1995, Kurucz CD-ROM, 23 (Cambridge: HarvardSmithonian Center for Astrophysics)

\section{FERENCES}

Lawler, J. E., Bonvallet, G., \& Sneden, C. 2001a, ApJ, 556, 452

Lawler, J. E., Wickliffe, M. E., Cowley, C. R., \& Sneden, C. 2001b, ApJS, 137,341

Lawler, J. E., Wickliffe, M. E., Den Hartog, E. A., \& Sneden, C. 2001c, ApJ, 563,1075

Lucatello, S., Gratton, R., Cohen, J. G., Beers, T. C., Christlieb, N., Carretta, E., \& Ramírez, S. 2003, AJ, 125, 875

McWilliam, A., Preston, G. W., Sneden, C., \& Searle, L. 1995a, AJ, 109, 2757

McWilliam, A., Preston, G. W., Sneden, C., \& Shectman, S. 1995b, AJ, 109, 2736

McWilliam, A. 1998, AJ, 115, 1640

Noguchi, K., et al. 2002, PASJ, 54, 855

Norris, J. E., Bessell, M. S., \& Pickles, A. J. 1985, ApJS, 58, 463

Norris, J. E., Ryan, S. G., \& Beers, T. C. 1996, ApJS, 107, 391

. 1997, ApJ, 489, L169 2001, ApJ, 561, 1034

Preston, G. W., \& Sneden, C. 2001, AJ, 122, 1545

Qian, Y.-Z., \& Wasserburg, G. J. 2001, ApJ, 552, L55 . 2002, ApJ, 567, 515

Rossi, S., Beers, T. C., \& Sneden, C. 1999, in ASP Conf. Ser. 165, The Third Stromlo Symp., The Galactic Halo, ed. B. K. Gibson, T. S. Axelrod, \& M. E. Putman (San Francisco: ASP), 268

Ryan, S. G., Norris, J. E., \& Beers, T. C. 1996, ApJ, 471, 254

Schatz, H., Toenjes, R., Pfeiffer, B., Beers, T. C., Cowan, J. J., Hill, V., \& Kratz, K.-L. 2002, ApJ, 579, 626

Sneden, C., Cowan, J. J., Ivans, I. I., Fuller, G. M., Burles, S., Beers, T. C., \& Lawler, J. E. 2000, ApJ, 533, 139

Sneden, C., McWilliam, A., Preston, G. W., Cowan, J. J., Burris, D. L., \& Armosky, B. J. 1996, ApJ, 467, 819

Sneden, C., et al. 2003, ApJ, 591, 936

Truran, J. W., Cowan, J. J., Pilachowski, C. A., \& Sneden, C. 2002, PASP, 114,1293

Van Eck, S., Goriely, S., Jorissen, A., \& Plez, B. 2001, Nature, 412, 793

Wanajo, S., Itoh, N., Ishimaru, Y., Nozawa, S., \& Beers, T. C. 2002, ApJ, 577,853

Wanajo, S., Tamamura, M., Itoh, N., Nomoto, K., Ishimaru, Beers, T. C., \& Nozawa, S. 2003, ApJ, 593, 968

Westin, J., Sneden, C., Gustafsson, B., \& Cowan, J. J. 2000, ApJ, 530, 783 